\title{
Grain boundary characteristics of Fe-based superconductors
}

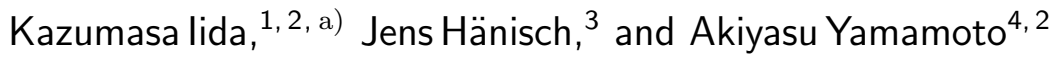

1) Department of Materials Physics, Nagoya University, Furo-cho, Chikusa-ku, Nagoya 464-8603, Japan

2) JST CREST, Sanbancho 5, Chiyoda-ku, Tokyo 102-0075, Japan

3) Institute for Technical Physics, Karlsruhe Institute of Technology, Hermann-von-Helmholtz-Platz 1, 76344 Eggenstein-Leopoldshafen, Germany

4) Department of Applied Physics, Tokyo University of Agriculture and Technology, 2-24-16 Naka-cho, Koganei-shi, Tokyo 184-8588, Japan

(Dated: 5 March 2022) 
Understanding the nature of grain boundary (GB) characteristics in combination with creating low-energy GBs by modifying the processing conditions, so-called GB engineering, is of great importance for controlling and reducing the defect density, leading to improved functionalities of polycrystalline metals and ceramics. For superconductors particularly, including both low- and high-temperature superconductors, GB engineering has been developed to improve especially the critical current densities, $J_{\mathrm{c}}$, across these GBs. The intrinsic physical properties of a given superconductor such as the coherence length, the order parameter symmetry, and their anisotropies would determine the strategy of GB engineering. In this topical review, we present an overview of the GB characteristics and GB engineering of Fe-based superconductors (FBS) in the form of polycrystalline bulks and wires, and thin films with application potential, e.g. for high-field magnet wires. Prior to the FBS, GB engineering of the cuprates and $\mathrm{MgB}_{2}$ are also briefly covered.

a)Electronic mail: iida@mp.pse.nagoya-u.ac.jp 


\section{INTRODUCTION}

Boundaries between adjacent crystallites of typically differing orientation and/or composition are called grain boundaries, where the atomic arrangement is in disorder. A grain boundary (GB) with a high interfacial energy (i.e. usually two adjacent grains having a large misorientation angle) is often the origin of macroscopic defects such as cracks and erosion damage, and these defects propagate along the GBs. On the other hand, GBs with a low interfacial energy [e.g. low-angle GBs and coincidence boundaries like twin boundaries] are hardly the origin of macroscopic defects. In low-angle GBs, dense dislocation arrays are formed with a spacing $D$ given by Frank's formula:

$$
D=\left(\frac{b}{2}\right) / \sin \left(\frac{\theta_{\mathrm{GB}}}{2}\right) \sim \frac{b}{\theta_{\mathrm{GB}}}
$$

where $b=|\vec{b}|$ is the norm of the Burgers vector (for symmetric [001]-tilt GBs in bicrystal films of e.g. cuprates and pnictides as discussed in this review, $|\vec{b}|$ is the in-plane lattice parameter) and $\theta_{\mathrm{GB}}$ is the misorientation angle between the two adjacent grains.

For both low- and high-temperature superconductors (LTS and HTS), GBs affect their properties: For instance, macroscopic defects (cracks) impede the supercurrent flow. In this case, GBs are detrimental defects to the critical current density $J_{c}$ and, hence, should be avoided.

For cuprates, not only macroscopic but also microscopic defects are problematic due to the short coherence length $\xi$ (less than $2 \mathrm{~nm}$ for $a b$-plane) ${ }^{1}$ and the anisotropic superconducting order parameter $(d \text {-wave })^{2}$. If the strained regions around the dislocations within the GB touch each other, the low-angle GB turns into a high-angle GB. This angle is around $10^{\circ} \sim 15^{\circ}$. However, in cuprates a wider region around the centre of the dislocation is perturbed electronically and the inter-grain $J_{\mathrm{c}}$ starts to decrease exponentially already for $\theta_{\mathrm{GB}}<10^{\circ}$. Also when 
the distance between strained regions is smaller or only slightly larger than the value of $\xi$ within the GB plane, the superconducting order parameter is depressed at the GB and, hence, the super-current flow is impeded. Additionally, bending of the electronic bands at GBs deplete the charge carrier density ${ }^{3}$, which seriously decreases the superconducting transition temperature $T_{\mathrm{c}}$. Hence, to achieve large $J_{c}$ for cuprates, the crystallites should not only be aligned out-of-plane because $J_{\mathrm{c}}$ is anisotropic but also be aligned in-plane within the misorientation angle $\theta_{\mathrm{GB}}$, where the distance between strained regions is larger than $\xi_{a b}\left(\xi_{a b}\right.$ : in-plane coherence length) is satisfied. On the other hand, for LTS and $\mathrm{MgB}_{2}$ with large coherence length (e.g. $5 \sim 6 \mathrm{~nm}$ ) and $s$-wave symmetry (for $\mathrm{MgB}_{2}$ anisotropic $s$ wave), GBs are able to pin vortices in the mixed state, and especially also GBs with rather large angles. Therefore, increasing the GB densities by reducing the grain size usually leads to improved in-field $J_{\mathrm{c}}$ properties.

As stated above, the symmetry of superconducting order parameter and the coherence length mainly govern the $J_{c}$ characteristics at GBs for a given superconductor. Hence, for both LTS and HTS, understanding the nature of GB characteristics in combination with modifying the processing conditions, so-called GB engineering, is of great importance for reducing and/or controlling the defect density to tune the superconducting properties.

The Fe-based superconductors (FBS) have similar physical properties as the cuprates, for instance a short coherence length due to the small carrier concentration and a low Fermi velocity ${ }^{4}$. Hence, FBS also seem to share the same GB issues as the cuprates. However, $J_{\mathrm{c}}$ across GBs in FBS is not as severely reduced due to several features, which is reviewed in this article. Many reviews of the synthesis of FBS in the form of bulk samples and thin films, and of their physical properties have been published to date. Additionally, excellent review articles concerning bicrystals and GBs in cuprates have already been published $\frac{3516}{3}$, however, only a few on GB issues for FBS have been published (e.g.7 and [8). 
In the following sections, we present an overview of the GB characteristics and GB engineering of the FBS in the form of polycrystalline bulks and wires as well as thin films with application potential, e.g. for high-field magnet wires and compact bulks. In section II, GB engineering of cuprates will be briefly summarised on the basis of the GB characteristics. Section III will describe how GBs affect the superconducting properties of $\mathrm{MgB}_{2}$. These sections II and III are relevant for understanding GB issues of FBS. Section IV will present the experimental reports on the misorientation angle dependence of the inter-grain $J_{\mathrm{c}}$ for various FBS films grown on symmetric [001]-tilt bicrystal substrates. In section V, GB engineering of FBS will be presented in the forms of both thin films and polycrystalline samples. The former part will describe how small misorientation angles affect the transport $J_{\mathrm{c}}$ of various FBS films deposited on technical substrates used for the second-generation coated conductors. The latter will present how to improve the superconducting properties of polycrystalline bulk and wire samples. Finally, we will discuss which kind of experiments are desired for better understanding the GB characteristics and summarise the review.

\section{GRAIN BOUNDARY ENGINEERING OF CUPRATES}

Thanks to $T_{\mathrm{c}}$ values higher than the boiling point of liquid nitrogen, the discovery of cuprates gave a significant excitement on our community. However, some material scientists were pessimistic about cuprates, since polycrystalline $R E \mathrm{Ba}_{2} \mathrm{Cu}_{3} \mathrm{O}_{7}$ ( $R E$ : rare earth elements, $\left.R E \mathrm{BCO}\right)$ was able to carry only $\sim 100 \mathrm{~A} / \mathrm{cm}^{2}$ even at $4.2 \mathrm{~K}^{9}$, which is far below the level needed for applications. A fundamental problem is that GBs with misorientation angle greater than $3^{\circ}$ (this angle is called the critical angle, $\theta_{c}$ ) constitute weak links. Figure 1 exemplifies the misorientation angle dependence of $J_{\mathrm{c}}$ for YBCO and Ag-doped YBCO grown on [001]-tilt symmetric $\mathrm{SrTiO}_{3}$ bicrystal substrates 10 . Above $\theta_{\mathrm{c}}$, the angle 


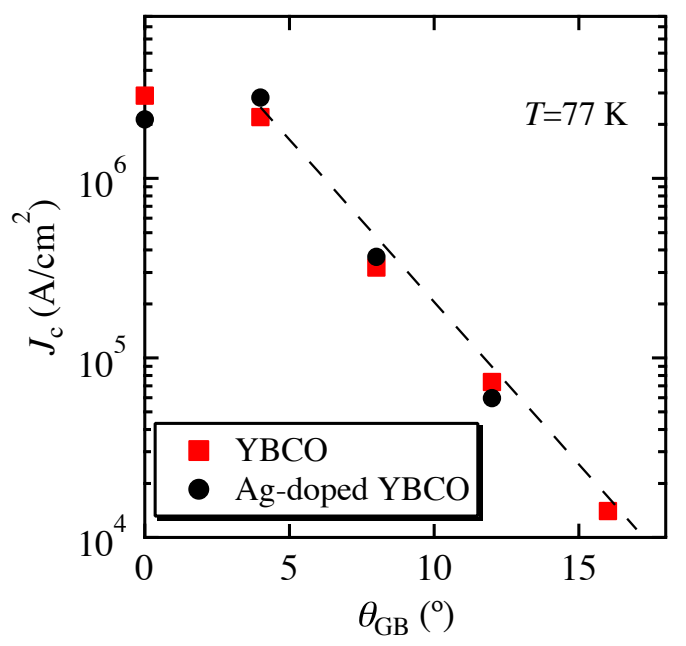

FIG. 1. Critical current density at $77 \mathrm{~K}$ of undoped and Ag-doped $\mathrm{YBa}_{2} \mathrm{Cu}_{3} \mathrm{O}_{7}$ (YBCO) films grown on $\mathrm{SrTiO}_{3}$ [001]-tilt bicrystals of different misorientations. The data are taken from 10. The dashed line is a fit by eq. (2).

dependence of inter-grain $J_{\mathrm{c}}\left(J_{\mathrm{c}}^{\mathrm{GB}}\right)$ is empirically described by

$$
J_{\mathrm{c}}^{\mathrm{GB}}\left(\theta_{\mathrm{GB}}\right)=J_{\mathrm{c}}^{\mathrm{G}} \exp \left(-\frac{\theta_{\mathrm{GB}}-\theta_{\mathrm{c}}}{\theta_{0}}\right)
$$

where $J_{\mathrm{c}}^{\mathrm{G}}$ is the intra-grain critical current density and $\theta_{0}$ is the characteristic angle ${ }^{10}$. The data were well described by the equation above with $\theta_{\mathrm{c}}=4^{\circ}$ and $\theta_{0}=2.4^{\circ}$. Also for other cuprates (e.g. $\mathrm{Nd}_{1.85} \mathrm{Ce}_{0.15} \mathrm{CuO}_{4-y}{ }^{11}$ and $\mathrm{Bi}_{2} \mathrm{Sr}_{2} \mathrm{Ca}_{2} \mathrm{Cu}_{3} \mathrm{O}_{10+\delta}{ }^{12}$ ) inter-grain $J_{\mathrm{c}}$ decreases exponentially with misorientation angle.

Local strain $\frac{13}{15}$ and bending of the electronic band structure ${ }^{\frac{16}{16}}$ resulting from the low carrier density combined with the large dielectric constant reduce the charge carrier density around GBs. As a result, dislocations or whole GB planes 
turn into antiferromagnetic insulators (i.e. Mott insulator).

Several models for the microscopic understanding of the transport properties across the GBs for YBCO have been proposed to date. Gurevich and Pashitskii concluded that the misorientation-angle dependence of inter-grain $J_{\mathrm{c}}$ is mainly determined by the progressive decrease in the cross-sectional area of superconductivity, which is induced by local strain $\frac{13}{\text {. T }}$. They calculated the critical angle $\theta_{\mathrm{c}}$ to be around $3^{\circ}-5^{\circ}$ at $77 \mathrm{~K}$, which is in good agreement with the experimental data. The progressive decrease in superconductivity around GBs is due to the shift of the Fermi level. The Fermi level of the GB regions having a thickness of the order of the Thomas-Fermi screening length lifts up around $5-10 \mathrm{meV}$ due to an excess of ion charge. Such small shifts are strong enough for a phase transition from superconductivity to antiferromagnetism to occur. This model explained the misorientation-angle dependence of inter-grain $J_{\mathrm{c}}$ for the low-angle regime. However, the regions of enhanced strain and possible off-stoichiometries of adjacent dislocations touch and overlap for misorientation angles larger than $\sim 10^{\circ}$ and, hence, the model cannot be applied for this angular regime. It is worth mentioning that the symmetry of the superconducting order parameter (isotropic $s$ - and anisotropic $d$-wave) seems not to affect the angle dependence of $J_{\text {c }}$ near the critical angle.

Another calculation based on the bond contraction model showed that the pairing is weakened by the tensile strain surrounding edge dislocations $\frac{15}{15}$. This explains the exponential decay of inter-grain $J_{\mathrm{c}}$. Kasatkin et al. calculated the inter-grain $J_{\text {c }}$ for [001]-tilt GBs on the assumption of the periodic pinning potential along the GBs $\frac{17}{17}$. They calculated the inter-grain $J_{\mathrm{c}}$ as a function of the in-plane coherence length and the norm of the Burgers vector, $b$, (as stated before, this value corresponds to the in-plane lattice parameter). In this model, the critical angle increases for decreasing ratio of $\xi_{a b} / b$. The results agree well with the experimental data for YBCO bicrystals with low misorientation angles. However, as pointed out 
by the authors, this model cannot explain the larger critical angle of FBS.

For arbitrary misorientation-angles, the inter-grain $J_{\mathrm{c}}$ has been calculated by Graser et al 18 . They initially simulated the atomic arrangement of YBCO at grain boundaries using a molecular dynamics procedure. Then a tight-binding model including the $d_{x^{2}-y^{2}}$ wave symmetry with parameters based on the atomic arrangement determined before was constructed for calculation. This calculation showed also good agreement with the experimental results.

As stated above, GB regions turn into insulating layers, which cannot be explained by the $d_{x^{2}-y^{2}}$ wave symmetry of the order parameter alone as pointed out by Schmehl et al $\underline{19}$. The product of the grain-boundary normal state resistance $R_{\mathrm{n}}$ and the grain boundary area $A$ is in the range $10^{-9} \leq R_{\mathrm{n}} A \leq 10^{-8} \Omega \mathrm{cm}^{2}$ at $4.2 \mathrm{~K}$ for $\operatorname{cuprates}^{20}$, which is one or two orders of magnitude higher than for Fe-based superconductors. To overcome these problems, Schmehl et al. tried to increase the charge carrier density by partially substituting $\mathrm{Y}^{+3}$ with $\mathrm{Ca}^{+2}$ in $\mathrm{YBCO}^{19}$. As a result, the inter-grain $J_{\mathrm{c}}$ for [001]-tilt $\mathrm{Y}_{0.7} \mathrm{Ca}_{0.3} \mathrm{Ba}_{2} \mathrm{Cu}_{3} \mathrm{O}_{7-\delta}$ bicrystal films with $\theta_{\mathrm{GB}}=24^{\circ}$ was enhanced by a factor of around 8 at $4.2 \mathrm{~K}$ compared to non-doped YBCO. Indeed, $E(J)$ curves at the same reduced temperature $\left(t=T / T_{\mathrm{c}}=0.85\right)$ for Ca-doped YBCO fabricated on [001]-tilt bicrystal substrate with $\theta_{\mathrm{GB}}=5^{\circ}$ did not show the non-ohmic linear differential behavior (i.e. the region where electric field $E$ increases linearly with the current density $J$ ) known from pure YBCO 21 . Nonohmic linear differential behavior is caused by viscous flux flow along the grain boundaries $^{22}$ and hence a clear indication that $J_{\mathrm{c}}$ is limited by GBs. Although Ca-doping mitigates the reduction of inter-grain $J_{c}$, the superconducting transition temperature is decreased to the range of $68 \leq T_{\mathrm{c}} \leq 79 \mathrm{~K}$, when $\mathrm{Ca}$ is doped not only in the GB region but also in the adjacent grains. In order to dope Ca only locally whilst grains are kept high- $T_{\mathrm{c}}$, Hammerl et al. fabricated a heterostructure of $\mathrm{YBCO} / \mathrm{Y}_{1-x} \mathrm{Ca}_{x} \mathrm{Ba}_{2} \mathrm{Cu}_{3} \mathrm{O}_{7-\delta}$ on $24^{\circ}$ [001]-tilt $\mathrm{SrTiO}_{3}$ bicrystal ${ }^{23}$. As a result, Ca preferentially diffused in the GB region and thereby doped the sample locally 
at the GB. The resultant films showed a self-field $J_{\mathrm{c}}$ as high as $3.3 \times 10^{5} \mathrm{~A} / \mathrm{cm}^{2}$ at $77 \mathrm{~K}$, which is almost the same level as for $7^{\circ}$ bicrystal films.

Another possible method of increasing the carrier concentration around GBs has been attempted recently by Fâte and Senatore by employing YBCO-based ionic-liquid gate transistors on $8^{\circ}$ [001]-tilt $\mathrm{SrTiO}_{3}$ bicrystal substrates 24 . Such an electric double layer transistor is a powerful tool to inject a large carrier density of $\sim 8 \times 10^{14} \mathrm{~cm}^{-2}$ at the material's surface ${ }^{25}$. The authors concluded that the level of carrier density by electrostatic doping was comparable to that of Cadoping mentioned above. A similar attempt on tuning GB properties by an electric double layer transistor device has been reported by Hassan and Wimbush on $\mathrm{La}_{1.85} \mathrm{Sr}_{0.15} \mathrm{CuO}_{4}$ by investigating the resistive transition 26 .

Another kind of GB engineering is the elimination of high-angle GBs. For this purpose, Jin et al. invented a melt-process for YBCO wires 27 . The resultant sample had a density of $6.2 \mathrm{~g} / \mathrm{cm}^{3}$, which is $98 \%$ of the theoretical density of YBCO. The self-field $J_{\mathrm{c}}$ of this melt-processed YBCO was almost doubled compared to sintered samples, however, $J_{\mathrm{c}}$ was decreased sharply by small magnetic fields of a few hundred gauss since high-angle GBs were not removed completely.

To eliminate high-angle GBs or at least minimise their density in $R E B C O$ bulk superconductors, the top-seeded-melt-growth process has been developed to grow quasi-single crystals 28 , which means that they contain secondary phase particles within the superconducting matrix and, therefore, are not true single crystals. $R E B C O$ bulks can trap large magnetic flux densities, and this ability is determined by $M=N J_{\mathrm{c}} r$, where $M$ is the volume magnetization, $N$ is a geometrical constant, and $r$ is the diameter of the circulating supercurrent. Hence, a general processing aim in the fabrication of bulk $R E \mathrm{BCO}$ is the production of large-grain, weak-link-free samples with high $J_{\mathrm{c}}$. To date, the largest trapped field of $17.6 \mathrm{~T}$ in bulk was recorded at $26 \mathrm{~K}$ in a stack of two GdBCO bulks with $25 \mathrm{~mm}$ in diameter 29 (Stacks of high- $T_{\mathrm{c}}$ tape showed a similarly high trapped field of $17.7 \mathrm{~T}$ at $8 \mathrm{~K}^{30}$ ). 
Now several companies have commercialised REBCO bulk superconductors. Applications by employing REBCO bulk superconductors are well summarised in ref.31.

Similar to REBCO bulks fabricated by the top-seeded melt growth process, epitaxial thin films on single-crystal substrates do not contain high-angle grain boundaries. Additionally, growth-related defects such as screw dislocations ${ }^{32}$ and stacking faults that work as strong pinning centers result in high $J_{\mathrm{c}}$ values of more than $1 \mathrm{MA} / \mathrm{cm}^{2}$ at $77 \mathrm{~K}$. However, the question arose how to fabricate singlecrystal-like $R E B C O$ films in long lengths, since a powder-in-tube method is not applicable to fabricating $R E \mathrm{BCO}$ wires. This issue was solved by the development of technical substrates that have been used for the 2nd generation HTS wires, the REBCO coated conductors. To date, mainly two kinds of technical substrates have been developed. One is the preparation of textured templates on Hastelloy C-276 tapes by ion beam assisted deposition (IBAD) ${ }^{33}$. IBAD yields biaxially textured buffer layers for epitaxial growth of $R E B C O$. The other is depositing epitaxial buffer layers on textured NiW tapes, which are thermo-mechanically treated (rolling-assisted biaxial texture, RABiTS) 34 . Thanks to the recent development of texture quality of those templates, the mean grain mis-orientation of $R E B C O$ lies in the range of $3-5^{\circ}$. Now tapes with high critical currents $I_{\mathrm{c}}$ and high uniformity of $\sim 1 \mathrm{~km}$ length are being fabricated, and several companies have commercialised $R E B C O$ coated conductors. Table 1 summarises GB engineering for $R E B C O$ in various forms. As stated above, all methods are based on physical properties of $R E B C O$. Hence, it is important to reveal the intrinsic physical properties of superconductors to determine the strategy for tackling GB issues. 
TABLE I. GB engineering for $R E B C O$ in various forms.

\begin{tabular}{|c|c|c|}
\hline Materials form & Methods & Refs. \\
\hline Bulks & Employing melt-process, top-seeded-melt-growth & 27 and 28 \\
\hline Thin films & Ca-doping, electrostatic carrier doping & 19, 21, 23, and 24, \\
\hline Tapes & Employing textured templates & 33 and 34 \\
\hline
\end{tabular}

\section{GRAIN BOUNDARY ENGINEERING OF $\mathrm{MGB}_{2}$}

$\mathrm{MgB}_{2}$ is a simple binary, metallic superconductor expected to be used at intermediate temperatures. Albeit $\mathrm{MgB}_{2}$ has a rather high $T_{\mathrm{c}}$ of $39 \mathrm{~K}$ and electromagnetic anisotropy of 2-5 $\frac{35}{3}$, GBs do not show the weak-link limitation of transport current $^{36}$ due to the large coherence length and anisotropic $s$-wave order parameter symmetry. Soon after the discovery of superconductivity in $\mathrm{MgB}_{2}$, high $J_{\mathrm{c}}$ was reported in untextured, polycrystalline wires produced by a powder-in-tube (PIT) process $\frac{37-40}{40}$ owing to its transparent GBs. Like in other intermetallic compound superconductors, GBs belong to the predominant flux pinning centers in $\mathrm{MgB}_{2} \stackrel{4145}{4}$. As shown in fig.2, a strong correlation between $J_{\mathrm{c}}$ and inverse grain size was observed ${ }^{42}$. Therefore, increasing the area density of GBs is an effective strategy to improve $J_{\mathrm{c}}$. On the other hand, $\mathrm{GBs}$ in $\mathrm{MgB}_{2}$ can be easily covered with impurity phases, such as $\mathrm{MgO}$ and amorphous oxides. These insulating particles and/or wetting layers at GBs act as transport current limiters and reduce the connectivity, which deteriorates the inter-grain $J_{\mathrm{c}}$ significantly. In this section, recent activities on $\mathrm{GB}$ engineering of $\mathrm{MgB}_{2}$ bulk samples, namely, controlling GB structure by doping/processing and enhancing GB density by grain size refinement and densification, are briefly introduced.

Refinement of $\mathrm{MgB}_{2}$ grains has been extensively studied with various methods, including the use of fine boron powder ${ }^{46}$, mechanical refinement by milling $47 \sqrt[40]{50}$, low-temperature heat processing $\sqrt[5155]{55}$, and impurity doping with $\mathrm{Ni}{ }^{56}, \mathrm{Cu}^{57} \sqrt[60]{60}$, $\mathrm{Ag}^{6162}, \operatorname{In}{ }^{63}$, and $\mathrm{Hf}^{64}$. Ma et al. reported that lamellar $\mathrm{MgB}_{2}$ grains can be 
formed with $\mathrm{Mg}-\mathrm{Cu}$ liquid, and these lamellar $\mathrm{MgB}_{2}$ grains are much better connected than the $\mathrm{MgB}_{2}$ grains of typical morphology 60 .

Another strategy for increasing the GB density per unit volume is densification, which also contributes to an improvement in connectivity by decreasing porosities. As densification techniques for bulk materials, high pressure synthesis and the magnesium diffusion method are commonly employed. To date, disk-shaped $\mathrm{MgB}_{2}$ bulks prepared by a hot-pressing technique have been prepared by many groups $\underline{65} \underline{68}$, and the resultant trapped field reached $3 \mathrm{~T}^{65}$. Nearly fully dense $\mathrm{MgB}_{2}$ bulks can be obtained by spark plasma sintering in a relatively short time (less than an hour) 69 . The magnesium diffusion/infiltration technique is known

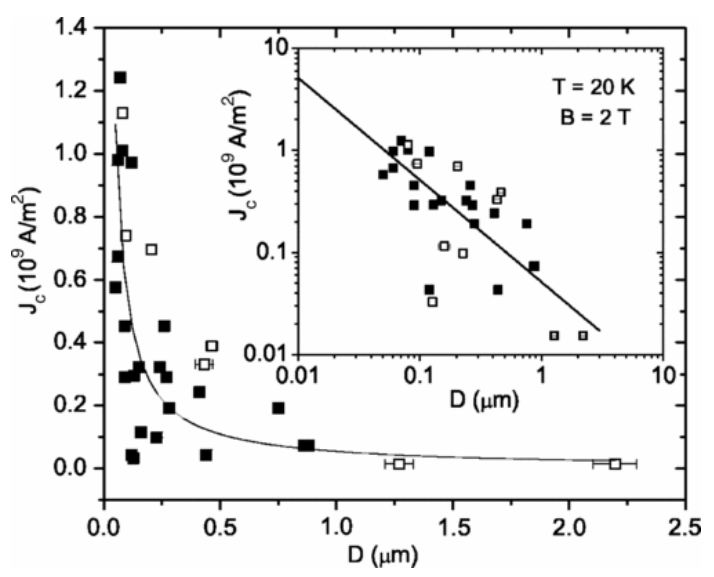

FIG. 2. Grain size $(D)$ dependence of $J_{\mathrm{c}}$ for dense $\mathrm{MgB}_{2}$ samples. The line fits to the $1 / D$ dependence. The open symbols are non-doped samples and the solid symbols are doped samples. Reprinted with permission from 42 . Copyright (2017) by the American Physical Society. 
as an alternative way to obtain dense $\mathrm{MgB}_{2}$ wires/bulks without applying high pressure during heating ${ }^{75}$.79]. Bhagurkar et al. reported nearly fully dense undoped and carbon-doped $\mathrm{MgB}_{2}$ bulks fabricated by the infiltration and growth process 80.82 . High trapped fields of $\sim 3 \mathrm{~T}$ have been measured at $5 \mathrm{~K}$ at the center of a stack of two bulk $\mathrm{MgB}_{2}$ samples fabricated using this technique ${ }^{80}$. Owing to the progress in the technologies of producing relatively large, dense $\mathrm{MgB}_{2}$ bulks, efforts in new bulk application fields, including magnetic shielding ${ }^{83}$, magnetic levitation $\sqrt{84}$, and biomedical applications 31 , as well as permanent-magnet applications have begun.

\section{IV. $\theta_{\mathrm{GB}}$ DEPENDENCE OF INTER-GRAIN $J_{\mathrm{c}}$ FOR FE-BASED SUPERCONDUCTING BICRYSTAL FILMS}

\section{A. $\mathrm{NdFeAs}(\mathrm{O}, \mathrm{F})$}

In the early stage of FBS research, $\operatorname{LaFeAs}(\mathrm{O}, \mathrm{F})^{85186}, \mathrm{SmFeAsO}_{0.85}, \mathrm{Nd}-$ $\mathrm{FeAs}(\mathrm{O}, \mathrm{F})^{87}$, and $\operatorname{SmFeAs}(\mathrm{O}, \mathrm{F})^{88}$ exhibited electromagnetic granularity similar to the cuprates. On the other hand, clear Meissner state due to bulk diamagnetism was observed in polycrystalline bulk $\operatorname{LnFeAs}(\mathrm{O}, \mathrm{F})(L n$ : lanthanoide) samples by magneto-optical imaging at a magnetic field below the lower critical field $H_{\mathrm{c} 1} 89$. Moreover, the roof top pattern was observed in the remanent magnetic field distribution image of the field-cooled sample, suggesting the macroscopic critical state of the whole bulk. Although $J_{\mathrm{c}}$ was higher than that of the untextured cuprates, it was still one or two orders of magnitude lower than $\mathrm{MgB}_{2}$, which does not show weak-link behaviour. Detailed microstructural analysis revealed that the early polycrystalline samples were multiphase composites containing impurities and structural defects, such as cracks and an amorphous wetting phase $\frac{87}{\text {. GBs }}$ of these samples were random and mixed with tilt and twist grain boundaries. Additionally, clean grain boundaries (i.e. no impurities between the grains) were 
also confirmed. To understand the effect of intrinsic and extrinsic factors on GB transport properties, bicrystal experiments on $\operatorname{LnFeAs}(\mathrm{O}, \mathrm{F})$ with single GB have been long desired. Under such circumstances, Omura et al. grew $\operatorname{NdFeAs}(\mathrm{O}, \mathrm{F})$ films on $\mathrm{MgO}$ bicrystal substrates with [001]-tilt GB of various misorientation angles by molecular beam epitaxy 90 . Here, $\operatorname{NdFeAs}(\mathrm{O}, \mathrm{F})$ thin films have been fabricated via a two-step process: Parent $\mathrm{NdFeAsO}$ is fabricated and subsequently a NdOF overlayer is deposited on NdFeAsO for F-doping 91 . Figure 3 shows the misorientation angle dependence of the GB transparency $J_{\mathrm{c}}^{\mathrm{GB}} / J_{\mathrm{c}}^{\mathrm{G}}\left(J_{\mathrm{c}}^{\mathrm{GB}}\right.$ : intergrain $J_{\mathrm{c}}, J_{\mathrm{c}}^{\mathrm{G}}$ : intra-grain $\left.J_{\mathrm{c}}\right)$ for $\operatorname{NdFeAs}(\mathrm{O}, \mathrm{F})$ fabricated on symmetric [001]-tilt $\mathrm{MgO}$ bicrystal substrates measured at $4.2 \mathrm{~K}$. The inter-grain $J_{\mathrm{c}}$ was reduced by $30 \%$ compared to the intra-grain $J_{\mathrm{c}}$ even at $\theta_{\mathrm{GB}}=6^{\circ}$, indicating that the critical angle for those samples may be less than $6^{\circ}$. However, the results show an extrinsic effect because $\operatorname{NdFeAs}(\mathrm{O}, \mathrm{F})$ was damaged by excess F. The $\operatorname{NdOF} / \operatorname{NdFeAs}(\mathrm{O}, \mathrm{F})$ bilayer is similar to $\mathrm{Y}_{0.7} \mathrm{Ca}_{0.3} \mathrm{Ba}_{2} \mathrm{Cu}_{3} \mathrm{O}_{7-\delta} / \mathrm{YBCO}$. As known from the work of $\mathrm{Y}_{0.7} \mathrm{Ca}_{0.3} \mathrm{Ba}_{2} \mathrm{Cu}_{3} \mathrm{O}_{7-\delta} / \mathrm{YBCO}$ bilayer ${ }^{23}$, Ca preferentially diffused through the GB and enriched Ca around the YBCO grains. Similarly, F diffused along the GB in $\operatorname{NdFeAs}(\mathrm{O}, \mathrm{F})$, however, the excess $\mathrm{F}$ deteriorated the $\operatorname{NdFeAs}(\mathrm{O}, \mathrm{F})$ layers due to the strong reactivity. To extract the intrinsic nature of the GBs in $\operatorname{NdFeAs}(\mathrm{O}, \mathrm{F})$, the damage around the GBs had to be minimised. Hence, the NdOF overlayer was deposited at $750^{\circ} \mathrm{C}$ instead of $800^{\circ} \mathrm{C}$ in order to suppress the excess $\mathrm{F}$ diffusion along the GB. With this method $J_{\mathrm{c}}^{\mathrm{GB}} / J_{\mathrm{c}}^{\mathrm{G}}$ is at nearly constant value of unity up to $8.5^{\circ}$ (Figure 3$)^{92}$. Beyond $8.5^{\circ}, J_{\mathrm{c}}^{\mathrm{GB}} / J_{\mathrm{c}}^{\mathrm{G}}$ decreases with $\theta_{\mathrm{GB}}$. Another distinct feature is that $J_{\mathrm{c}}^{\mathrm{GB}} / J_{\mathrm{c}}^{\mathrm{G}}$ is larger than 1 at $\theta_{\mathrm{GB}}=6^{\circ}$. A plausible reason for this phenomenon is GB pinning. This may explain the improvement of $J_{\mathrm{c}}$ for Co- and P-doped $\mathrm{BaFe}_{2} \mathrm{As}_{2}$ deposited on technical substrates ${ }^{93 \mid 94}$, which is discussed later.

Lowering the deposition temperature for the $\mathrm{NdOF}$ overlayer certainly improved the GB properties of $\mathrm{NdFeAs}(\mathrm{O}, \mathrm{F})$. However, both inter- and intra-grain $J_{\mathrm{c}}$ in selffield was of the order of $10^{5} \mathrm{~A} / \mathrm{cm}^{2}$, which is one order of magnitude lower than 


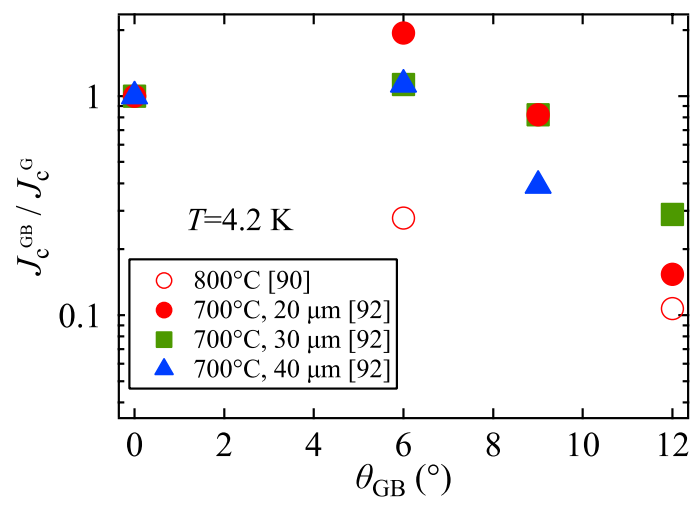

FIG. 3. Angle dependence of the GB transparency $J_{\mathrm{c}}^{\mathrm{GB}} / J_{\mathrm{c}}^{\mathrm{G}}$ at $4.2 \mathrm{~K}$ for $\operatorname{NdFeAs}(\mathrm{O}, \mathrm{F})$ bicrystal films with different deposition temperatures and thicknesses of the NdOF overlayer.

that of an ordinary $\operatorname{NdFeAs}(\mathrm{O}, \mathrm{F})$ (i.e. deposition temperature of $\mathrm{NdOF}$ is $800^{\circ} \mathrm{C}$ ). Additionally, damage to the GB region by $\mathrm{F}$ is inevitable despite lowering the deposition temperature of $\mathrm{NdOF}$. Therefore, studying F-free oxypnictide would be necessary. To date, polycrystalline $\operatorname{Ln}(\mathrm{Fe}, \mathrm{Co}) \mathrm{AsO}^{95}$ and epitaxial $\operatorname{SmFeAs}(\mathrm{O}, \mathrm{H})^{96}$ thin films are available and, therefore, studying the GB angle dependence of $J_{\mathrm{c}}$ of bicrystal films of those compounds would be reasonable.

\section{B. Co- and P-doped $\mathrm{BaFe}_{2} \mathrm{As}_{2}$}

Lee et al. in 2009 were the first to show possible weak-link behaviour of [001]-tilt GBs in $\mathrm{Ba}\left(\mathrm{Fe}_{1-x} \mathrm{Co}_{x}\right)_{2} \mathrm{As}_{2}$ films $\left(T_{\mathrm{c}}=20.5 \mathrm{~K}\right)$ grown by pulsed laser deposition on 
bicrystalline $\mathrm{SrTiO}_{3}$ substrates containing GBs with angles $\theta_{\mathrm{GB}}$ of $3^{\circ}, 6^{\circ}, 9^{\circ}$, and $24^{\circ}$ 97. Also the low-angle GBs with $\theta_{\mathrm{GB}}=6^{\circ}$ and $9^{\circ}$ showed weak-link behaviour in low-temperature laser scanning microscopy and magneto-optical imaging. This suggested a critical angle $\theta_{\mathrm{c}}<6^{\circ}$, which later turned out to be in contrast to the results reported by Katase, Hiramatsu et al. ${ }^{98 \mid 99}$ with a critical angle $\theta_{\mathrm{c}} \sim 9^{\circ}$ from $\mathrm{Ba}\left(\mathrm{Fe}_{1-x} \mathrm{Co}_{x}\right)_{2} \mathrm{As}_{2}$ films on $\mathrm{MgO}\left(T_{\mathrm{c}}=20.7 \mathrm{~K}\right)$ and LSAT $\left(T_{\mathrm{c}}=21.6 \mathrm{~K}\right)$ bicrystals. Presumably, an increased oxygen diffusion from $\mathrm{SrTiO}_{3}$ under ultra high vacuum conditions along the GB leads to a slightly broader region of reduced order parameter around the GB which may have caused the $J_{\mathrm{c}}$ reduction of the low-angle

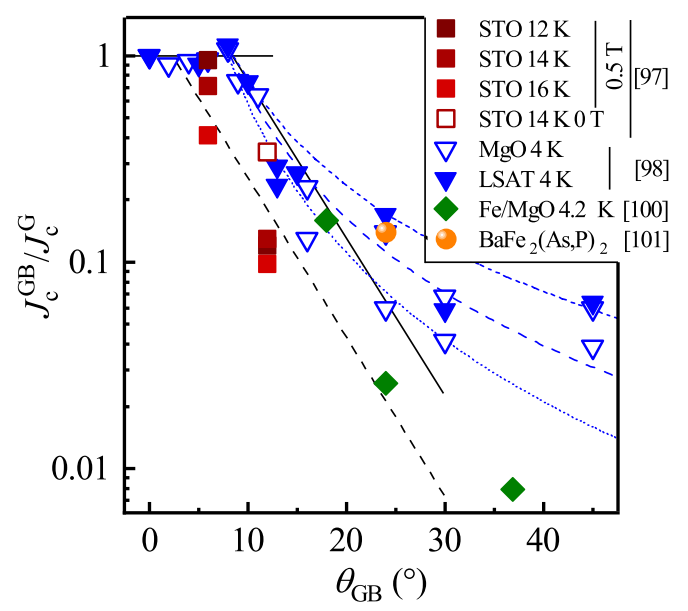

FIG. 4. Angle dependence of the GB transparency for $\mathrm{Ba}\left(\mathrm{Fe}_{1-x} \mathrm{Co}_{x}\right)_{2} \mathrm{As}_{2}$ bicrystal films on different substrates $\left[\left(\mathrm{LaAlO}_{3}\right)_{0.3}\left(\mathrm{Sr}_{2} \mathrm{TaAlO}_{6}\right)_{0.7}(\mathrm{LSAT}), \mathrm{SrTiO}_{3}\right.$ (STO) and Fe-buffered $\mathrm{MgO}(\mathrm{Fe} / \mathrm{MgO})]$ grown by pulsed laser deposition and a $\mathrm{BaFe}_{2}\left(\mathrm{As}_{1-x} \mathrm{P}_{x}\right)_{2}$ bicrystal film on $\mathrm{MgO}$ grown by molecular beam epitaxy. The lines are exponential (black) and power-law (blue) approximations of the data according to eqs. 2 and 3 , respectively. 
GB 97 . $T_{\mathrm{c}}$ was only minimally reduced across the $6^{\circ}$ junction on $\mathrm{SrTiO}_{3}$ by $0.2 \mathrm{~K}$ with sharp resistive transition. Nevertheless, the elevated applied fields $(500 \mathrm{~m} \mathrm{~T})$ and measurement temperatures $\left(12,14\right.$ and $16 \mathrm{~K}$ ) in ${ }^{97}$ should be regarded (data at low temperature and magnetic field are comparable to the ones reported in ${ }^{98 \mid 99}$, fig.(4). Later, Iida et al. investigated high-angle GBs in $\mathrm{Ba}\left(\mathrm{Fe}_{1-x} \mathrm{Co}_{x}\right)_{2} \mathrm{As}_{2}$ films grown on Fe/spinel-buffered $\mathrm{SrTiO}_{3}$ bicrystal substrates 100 . The critical angle was around $8^{\circ}$. The $J_{\mathrm{c}}$ reduction of the high-angle GBs, nevertheless, was 10 times larger than shown by Katase et al, which again is most likely due to defects in the GB region because of the oxygen diffusion from $\mathrm{SrTiO}_{3}$. Sakagami et al. showed $J_{\mathrm{c}}^{\mathrm{GB}}$ as high as $1 \mathrm{MA} / \mathrm{cm}^{2}$ at $4 \mathrm{~K}$ for $\theta_{\mathrm{GB}}=24^{\circ}$ for a $\mathrm{BaFe}_{2}\left(\mathrm{As}_{1-x} \mathrm{P}_{x}\right)_{2}$ film on an $\mathrm{MgO}$ bicrystal grown by molecular beam epitaxy 101 , which is due to the higher $T_{\mathrm{c}}$ and hence higher $J_{\mathrm{c}}^{\mathrm{G}}$ than for Co-doped $\mathrm{BaFe}_{2} \mathrm{As}_{2}$. Despite the low number of data points, $\theta_{\mathrm{c}}$ of $\mathrm{BaFe}_{2}\left(\mathrm{As}_{1-x} \mathrm{P}_{x}\right)_{2}$ is most likely also larger than for the cuprates. The $J_{\mathrm{c}}\left(\theta_{\mathrm{GB}}\right)$ dependence for GBs in Co- and P-doped $\mathrm{BaFe}_{2} \mathrm{As}_{2}$ as available in literature is summarised in Figure 4. Clearly, $J_{\mathrm{c}}^{\mathrm{GB}}$ remains at $J_{\mathrm{c}}^{\mathrm{G}}$ up to $\theta_{\mathrm{c}} \sim 9^{\circ}$. At higher angles, exponential decay of the GB transparency with a slope similar to that of the cuprates (dashed black line) is observed. $J_{c}^{\mathrm{GB}}$ is, on the other hand, nearly constant for $30^{\circ}$ and $45^{\circ}$. An alternative possible phenomenological description of the data beyond $\theta_{\mathrm{c}}$ is shown as blue dashed lines according to

$$
\frac{J_{\mathrm{c}}^{\mathrm{GB}}}{J_{\mathrm{c}}^{\mathrm{G}}}=\left(\frac{\theta_{\mathrm{GB}}}{\theta_{0}}\right)^{-n}
$$

with sets of parameters $\left(\theta_{0}, n\right)$ of $(8.5,1.7),(8.25,2.05)$, and $(8.0,2.4)$ for high, medium and low $J_{\mathrm{c}}$ values, respectively. Such a function might be worthwhile for testing in statistical simulation models of GB networks such as in ref. ${ }^{102}$, however the differences to the logarithmic fits may be small, especially for sharp textures.

Occasionally, pinning effects can be observed in single high-angle GBs, even though the absolute $J_{\mathrm{c}}$ value might not be higher than the intra-grain values. An example is found in ref. ${ }^{97}$ for the $24^{\circ} \mathrm{GB}$ junction of $\mathrm{Ba}\left(\mathrm{Fe}_{1-x} \mathrm{Co}_{x}\right)_{2} \mathrm{As}_{2}$ on $\mathrm{SrTiO}_{3}$. 
Despite the lower $J_{\mathrm{c}}$ values for all applied fields, a peak effect is visible near $5 \mathrm{~T}$ which can be attributed to pinning of vortices in the GB plane or surrounding region itself as well as by interaction with vortices in the grains nearby. In ref. 98 , this effect can be recognised as well but is not discussed there. High-angle GBs can also supply pinning centres indirectly: Fe nanoparticles preferably grow in or near such GBs, see ref. $\stackrel{103}{ }$, most likely because of higher diffusion rates in the grain boundaries or strain effects. $J_{\mathrm{c}}$ of the sample with these $45^{\circ} \mathrm{GBs}$ was increased in a wide range of field and temperature compared to a similar sample without $45^{\circ}$ misorientations with a pronounced $c$-axis peak due to the $c$-axis-oriented GBs and the slightly $c$-axis-elongated Fe nanoparticles.

\section{C. $\mathrm{Fe}(\mathrm{Se}, \mathrm{Te})$}

A self-field inter-grain $J_{\mathrm{c}}$ of around $10^{4} \mathrm{~A} / \mathrm{cm}^{2}$ at low temperatures for $\mathrm{Fe}(\mathrm{Se}, \mathrm{Te})$ of a $45^{\circ} \mathrm{GB}^{104}$, only 10 times lower than the intra-grain $J_{\mathrm{c}}$ (instead of $10^{4}$ as for the cuprates) suggested a $J_{\mathrm{c}}$ decrease with $\theta_{\mathrm{GB}}$ not as strong as for the cuprates. $J_{c}$ values being rather independent of the bridge width and the uniform Josephson current along the junction were attributed by Sarnelli et al. to the $s$-wave symmetry in these materials. Later, the full $J_{\mathrm{c}}\left(\theta_{\mathrm{GB}}\right)$ dependence with $\theta_{\mathrm{c}} \sim 9^{\circ}$ was measured by Si et al. ${ }^{105}$ and Sarnelli et al. $\stackrel{106}{ }$, see fig. 5. As Si et al. found, $J_{\mathrm{c}}\left(\theta_{\mathrm{GB}}\right)$ maintains $\sim 10^{5} \mathrm{~A} / \mathrm{cm}^{2}$ at $4.2 \mathrm{~K}$ for $\theta_{\mathrm{GB}}<7^{\circ}$ even in applied fields of around $10 \mathrm{~T}$, showing that these GBs are strong links. For $\theta_{\mathrm{GB}}>15^{\circ}$ the GBs showed significantly lower $J_{\mathrm{c}}$ values and a foot structure in the temperature dependence of the resistivity, $\rho(T)$, due to a larger distorted region near the GB. If intrinsic effects, such as thermally activated phase slippage $\frac{107}{10}$, may contribute in such samples with low $T_{\mathrm{c}}$ values, is still to be investigated. In contrast to NdFeAs $(\mathrm{O}, \mathrm{F})$ and $\mathrm{Ba}\left(\mathrm{Fe}_{1-x} \mathrm{Co}_{x}\right)_{2} \mathrm{As}_{2}$, the GB transparency also decreased below $\theta_{\mathrm{c}}$, and no increased values were found near $\theta_{c}$. However, further experiments in the 


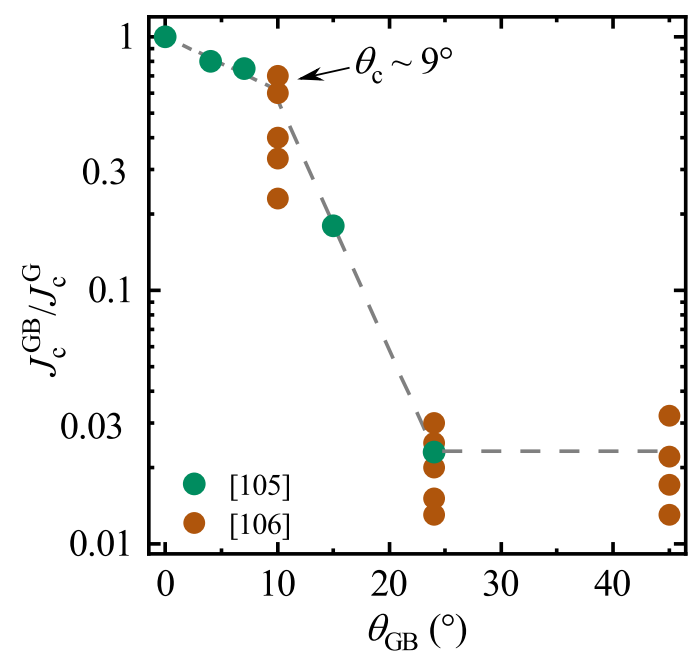

FIG. 5. Angle dependence of the self-field GB transparency for $\mathrm{Fe}(\mathrm{Se}, \mathrm{Te})$ bicrystal junctions at $4 \mathrm{~K}$.

vicinity of the critical angle are necessary to clarify this. The level of $J_{\mathrm{c}}^{\mathrm{GB}}$ is almost constant above $24^{\circ} 106$.

\section{GRAIN BOUNDARY ENGINEERING OF FE-BASED SUPERCONDUCTORS}

\section{A. Grain boundary networks in Fe-based superconducting films}

Long-length YBCO coated conductors are fabricated on polycrystalline templates, which are in- and out-of-plane textured. Hence, thin films on technical substrates (e.g. Hastelloy) contain many low-angle GBs, which provides a good platform for studying how low-angle GB networks affect $J_{\mathrm{c}}$. To date, most of 
the FBS coated conductors as proof-of-principle studies have been fabricated on metallic templates with biaxially textured $\mathrm{MgO}$ prepared by IBAD. Fe(Se,Te) has been also deposited on $\mathrm{CeO}_{2}$-buffered RABiTS. Here, we briefly review each FBS on technical substrates.

\section{1. $\operatorname{Ln} \mathrm{FeAs}(\mathrm{O}, \boldsymbol{F})$.}

So far, only $\operatorname{NdFeAs}(\mathrm{O}, \mathrm{F})$ has been grown on a technical substrate among LnFeAs $(\mathrm{O}, \mathrm{F})$. Iida, Hänisch et al. reported on the fabrication of $\mathrm{NdFeAs}(\mathrm{O}, \mathrm{F})$ on IBAD-MgO by molecular beam epitaxy 108 . Structural characterization by X-ray diffraction revealed some $45^{\circ}$ rotated grains, resulting in a low self-field $J_{\mathrm{c}}$ at $5 \mathrm{~K}$ of $70 \mathrm{kA} / \mathrm{cm}^{2}$ (corresponding to a reduced temperature of $t=T / T_{\mathrm{c}, 0}=0.135$ ), which is comparable to the $40 \mathrm{kA} / \mathrm{cm}^{2}$ at $4.2 \mathrm{~K}$ measured for partially textured PIT Sm$\mathrm{FeAs}(\mathrm{O}, \mathrm{F})$ tape $\frac{109}{}$. However, it was 1.5 times lower than $J_{\mathrm{c}}$ on $\mathrm{MgO}$ single crystal of $3 \mathrm{MA} / \mathrm{cm}^{2110}$. After optimisation of the deposition conditions for $\operatorname{NdFeAs}(\mathrm{O}, \mathrm{F})$ on IBAD-MgO, rotated grains were successfully removed, resulting in a record self-field $J_{\mathrm{c}}$ for this kind of films of around $2 \mathrm{MA} / \mathrm{cm}^{2} \underline{111}$.

\section{2. $\boldsymbol{B a}\left(\mathrm{Fe}_{1-x} \mathrm{Co}_{x}\right)_{2} \boldsymbol{A} s_{2}$.}

Already in 2011, $\mathrm{Ba}\left(\mathrm{Fe}_{1-x} \mathrm{Co}_{x}\right)_{2} \mathrm{As}_{2}$ films were grown on Fe-buffered IBAD$\mathrm{MgO}$ templates by pulsed laser deposition 112. The films were biaxially textured, and the crystalline quality of both Fe-buffer and superconducting layers with full width at half maximum values of $\Delta \phi_{\mathrm{Fe}} \sim 5^{\circ}$ and $\Delta \phi_{\mathrm{Ba} 122} \sim 5^{\circ}$ was comparable to that of the $\mathrm{MgO}\left(\Delta \phi_{\mathrm{MgO}} \sim 6^{\circ}\right)$. The $\mathrm{Ba}\left(\mathrm{Fe}_{1-x} \mathrm{Co}_{x}\right)_{2} \mathrm{As}_{2}$ coated conductor showed an onset $T_{\mathrm{c}}$ of $22 \mathrm{~K}\left(T_{\mathrm{c}, 0}=17.5 \mathrm{~K}\right)$, which was a relatively large transition width. Due to the poor crystalline quality, the self-field $J_{\mathrm{c}}$ of $0.1 \mathrm{MA} / \mathrm{cm}^{2}$ at $8 \mathrm{~K}(t=0.457)$ was 8-10 times lower than on single crystalline $\mathrm{MgO}$. This electro-magnetic granularity in $\mathrm{Ba}\left(\mathrm{Fe}_{1-x} \mathrm{Co}_{x}\right)_{2} \mathrm{As}_{2}$ coated conductors was later suppressed by employing 
a sharply in-plane textured $\mathrm{MgO}\left(\Delta \phi_{\mathrm{MgO}}=2.4^{\circ}\right)$, leading to a $J_{\mathrm{c}}$ level comparable to the film on single crystalline substrate ${ }^{113}$. Shortly after, Katase et al. grew $\mathrm{Ba}\left(\mathrm{Fe}_{1-x} \mathrm{Co}_{x}\right)_{2} \mathrm{As}_{2}$ on IBAD-MgO without a buffer layer $\underline{93}$. Thanks to the self-epitaxy effect, a sharp in-plane texture of $\Delta \phi_{\mathrm{Ba} 122}=3.2-3.5^{\circ}$ was reached irrespective of the template's $\Delta \phi_{\mathrm{MgO}}$ values.

Although $J_{\mathrm{c}}$ is limited by GBs in general, GBs in FBS also have advantages: a) The larger $\theta_{c}$ than for the cuprates (as reviewed above) allows for less-textured templates. b) GBs may contribute to flux pinning, resulting in high current carrying capabilities in magnetic field ${ }^{93}$ : $\mathrm{Ba}\left(\mathrm{Fe}_{1-x} \mathrm{Co}_{x}\right)_{2} \mathrm{As}_{2}$ films on IBAD-MgO of different in-plane full width at half maximum values $\left(\Delta \phi_{\mathrm{MgO}}=5.5^{\circ}, 6.1^{\circ}, 7.3^{\circ}\right)$ lead to $\mathrm{Ba}\left(\mathrm{Fe}_{1-x} \mathrm{Co}_{x}\right)_{2} \mathrm{As}_{2}$ texture spreads of $\Delta \phi_{\mathrm{Ba} 222}=3.1^{\circ}, 3.2^{\circ}$, and $3.5^{\circ}$. Apparently, the self-field $J_{\mathrm{c}}\left(1.2,1.6,3.6 \mathrm{MA} / \mathrm{cm}^{2}\right)$ is correlated to $\Delta \phi_{\mathrm{Ba} 122}$, which is due to $\theta_{\mathrm{GB}}$ of most GBs being below $\theta_{\mathrm{c}}$ (i.e. they are strong links) and due to an increasing dislocation density with misorientation spread. One of the films on IBAD-MgO $\left(\Delta \phi_{\mathrm{MgO}}=6.1^{\circ}\right)$ showing higher $J_{\mathrm{c}}(B \| c)$ values than a film on $\mathrm{MgO}$ single crystal for all fields at low and medium temperature and even higher than $J_{\mathrm{c}}(B \| a b)$ up to a temperature-dependent cross-over field supported this finding.

$\mathrm{Xu}$ et al. reported recently on the growth of $\mathrm{Ba}\left(\mathrm{Fe}_{1-x} \mathrm{Co}_{x}\right)_{2} \mathrm{As}_{2}$ on $\mathrm{SrTiO}_{3} / \mathrm{LaMnO}_{3}-$ buffered IBAD-MgO 114 . Almost isotropic $J_{\mathrm{c}}$ of 0.86 and $0.96 \mathrm{MA} / \mathrm{cm}^{2}$ at $9 \mathrm{~T}$ and for $B \| c$ and $B \| a b$, respectively, were recorded at $4.2 \mathrm{~K}$.

To understand the $J_{\mathrm{c}^{-}} B$ properties of FBS grown on technical substrates, Abrikosov-Josephson vortices should also be taken into account. Abrikosov vortices with Josephson cores, so-called Abrikosov-Josephson vortices, are present in low-angle $\mathrm{GBs}$ for $\mathrm{YBCO}$ 115/116. Although there are no reports on AbrikosovJosephson vortices in FBS, they might be present in GB networks having small misorientation angles. As has been reported by Palau et al. for cuprates 117 , the inter-grain $J_{\mathrm{c}}$ correlates with intra-grain $J_{\mathrm{c}}$ due to the magnetic interaction between Abrikosov-Josephson vortices at low-angle GBs and Abrikosov vortices 
in the grains. Hence, it is possible to improve the pinning potential of AbrikosovJosephson vortices by increasing the density of strong pinning centres in close vicinity of the GB region.

\section{3. $\boldsymbol{B a F e}_{2}\left(\boldsymbol{A} s_{1-x} \boldsymbol{P}_{x}\right)_{2}$.}

Sato et al. grew $\mathrm{BaFe}_{2}\left(\mathrm{As}_{1-x} \mathrm{P}_{x}\right)_{2}$ on IBAD-MgO with $\Delta \phi_{\mathrm{MgO}}=4^{\circ}$ and $8^{\circ} \underline{94}$ by pulsed laser deposition. Energy dispersive X-ray spectroscopy in TEM cross sectional images showed clean and homogeneous GBs. The grain size of $\sim 100 \mathrm{~nm}$ as well as dislocation arrays in the GB regions were seen in plane-view TEM images

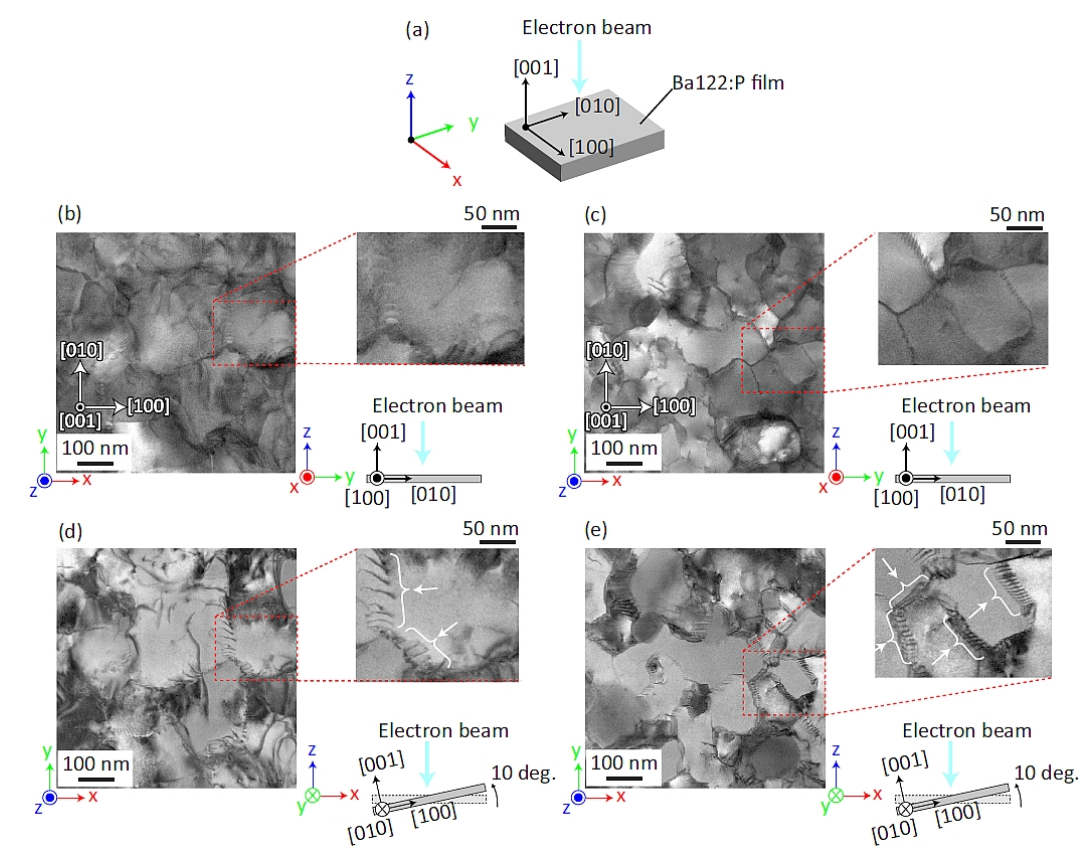

FIG. 6. Plane-view bright-field scanning TEM images of $\mathrm{BaFe}_{2}\left(\mathrm{As}_{1-x} \mathrm{P}_{x}\right)_{2}$ films on two IBAD templates with $\Delta \phi_{\mathrm{MgO}}=4^{\circ}$ and $8^{\circ}$. (a) Relationship between film orientation, incident electron beam, and global axes (X, Y, and Z). (b, c) Typical plane-view images by normal electron beam incidence for $\Delta \phi_{\mathrm{MgO}}=4^{\circ}$ (b) and $8^{\circ}$ (c). (d, e) Slanted-angle images (tilting by $10^{\circ}$ ) for visualizing dislocations at same areas of $(\mathrm{b}, \mathrm{c})$, respectively. The arrows in the top right image of $(\mathrm{d}, \mathrm{e})$ show arrays of dislocations. Reproduced with permission from Sato et al., Sci. Rep. 6, 36828 (2016). Copyright 2016 Spring Nature. 
(fig. 6). The crystalline quality of the film on the $4^{\circ}$ substrate was better than that of the film on $\mathrm{MgO}$ with $\Delta \phi_{\mathrm{MgO}}=8^{\circ}$, and its $T_{\mathrm{c}, 0}$ of $23 \mathrm{~K}$ was $4 \mathrm{~K}$ higher than for the $8^{\circ}$ substrate. Nevertheless, the film on the $8^{\circ}$ substrate showed a larger $c$-axis peak and 1.5 times higher $J_{\mathrm{c}}$ values than the $4^{\circ}$ template, which is due to the larger density of especially vertical defects related to the GB networks. Since it has a higher $J_{\mathrm{c}}$ and therefore also irreversibility line, the $4^{\circ}$ sample surpasses the $8^{\circ}$ sample at a temperature-dependent crossover field. However, the $8^{\circ}$ sample still shows the stronger $c$-axis peak. One of these samples on $8^{\circ}$ template was further investigated in high fields 118 . The field-dependent pinning force densities, $F_{\mathrm{p}}(H)$, analysed with the modified Dew-Hughes model, suggest that pinning is strongly dominated by surface pinning, i.e. on 2-dimensional defects in the matrix such as dislocation networks and full GBs. The $V(I)$ curves clearly showed a crossover between GB limitation (non-ohmic linear differential) at low fields and and pinning limitation (power law behaviour, representing flux creep effect) at high fields. Similar non-ohmic linear differential behaviour has been measured for $\mathrm{Ba}\left(\mathrm{Fe}_{1-x} \mathrm{Co}_{x}\right)_{2} \mathrm{As}_{2}$ with $45^{\circ}$ and (110) misoriented grains ${ }^{[19}$ and polycrystalline $\mathrm{SmFeAsO}_{0.85} \frac{120}{\text {. }}$.

A first $10 \mathrm{~cm}$ long $\mathrm{BaFe}_{2}\left(\mathrm{As}_{1-x} \mathrm{P}_{x}\right)_{2}$ tape was fabricated by pulsed laser deposition with a reel-to-reel system at a tape travelling speed of $6 \mathrm{~mm} / \mathrm{min}^{121}$, and its $T_{\mathrm{c}, 0}$ was reduced $\sim 17 \mathrm{~K}$, which is slightly low compared to the static samples $\left(T_{\mathrm{c}, 0} \sim 20 \mathrm{~K}\right)$. $I_{\mathrm{c}}$ was $0.47 \mathrm{~mA} / \mathrm{cm}$-width at $4.2 \mathrm{~K}$, corresponding to $J_{\mathrm{c}}=47 \mathrm{kA} / \mathrm{cm}^{2}$. For enhancing $T_{\mathrm{c}}$ and $J_{\mathrm{c}}, \mathrm{Fe}_{3} \mathrm{P} / \mathrm{BaFe}_{2}\left(\mathrm{As}_{1-x} \mathrm{P}_{x}\right)_{2}$ bilayers were fabricated on IBAD-MgO. This led to improved $T_{\mathrm{c}, 0}$ of $24 \mathrm{~K}$ and $I_{\mathrm{c}}$ of $975 \mathrm{~mA} / \mathrm{cm}$-width $\left(J_{\mathrm{c}}=175 \mathrm{kA} / \mathrm{cm}^{2}\right)$ at $4.2 \mathrm{~K}$. 


\section{4. $\mathrm{Fe}(\mathrm{Se}, \mathrm{Te})$.}

Si et al. were able to deposit high quality Fe(Se,Te) films on IBAD-MgO despite the relatively large mismatch of $9.5 \% \frac{122}{2}$. The in- and out-of-plane full width at half maximum values of $\mathrm{Fe}(\mathrm{Se}, \mathrm{Te})$ were $\Delta \phi_{\mathrm{Fe}(\mathrm{Se}, \mathrm{Te})}=4.5^{\circ}\left(<\theta_{\mathrm{c}}\right)$ and $\Delta \omega_{\mathrm{Fe}(\mathrm{Se}, \mathrm{Te})}=$ 3.5 . Since also [010]-tilt GBs in FBS probably have a similar $\theta_{\mathrm{c}}$ value, the GBs do not act as weak-links in these films. The lower $T_{\mathrm{c}, 0}$ of $11 \mathrm{~K}$ compared to single crystalline $\mathrm{LaAlO}_{3}$ substrate $(\sim 15 \mathrm{~K})$ can be attributed to the large misfit and reduces self-field $J_{\mathrm{c}}$ at $4 \mathrm{~K}$ to $0.2 \mathrm{MA} / \mathrm{cm}^{2}$. Xu et al. grew $\mathrm{Fe}(\mathrm{Se}, \mathrm{Te})$ films on $\mathrm{LaMnO}_{3} / \mathrm{IBAD}-\mathrm{MgO}$ 123. The $T_{\mathrm{c}}$ of $15.8 \mathrm{~K}$ was slightly enhanced due to the in-plane lattice compression. Whereas $\Delta \omega_{\mathrm{Fe}(\mathrm{Se}, \mathrm{Te})}=3.4^{\circ}$ was similar to the film in ref. ${ }^{[122}, \Delta \phi_{\mathrm{Fe}(\mathrm{Se}, \mathrm{Te})}=7.8^{\circ}<\theta_{\mathrm{c}}$ was larger. The self-field $J_{\mathrm{c}}$ at $4.2 \mathrm{~K}$ was $0.43 \mathrm{MA} / \mathrm{cm}^{2}$ nonetheless.

Not only IBAD-MgO templates but also $\mathrm{CeO}_{2}$-buffered RABiTS were used for the growth of $\mathrm{Fe}(\mathrm{Se}, \mathrm{Te})$ films ${ }^{124}$. The relevant lattice parameter of $\mathrm{CeO}_{2}$ is around $3.82 \AA$, hence close to the in-plane parameters of $\mathrm{Fe}(\mathrm{Se}, \mathrm{Te})$. Si et al. achieved films on RABiTS with a high $T_{\mathrm{c}}$ of $\sim 20 \mathrm{~K}$ and sharp resistive transition, which may be due to the small lattice mismatch and absence of intercalated Fe. Even though the in-plane full width at half maximum value was $\sim 6^{\circ}$, (corresponding to the underlying template of $\Delta \phi_{\mathrm{CeO} 2}=7^{\circ}$ ), a large self-field $J_{\mathrm{c}}$ of $1.5 \mathrm{MA} / \mathrm{cm}^{2}$ at $4.2 \mathrm{~K}$ was measured. According to the authors, the $\mathrm{CeO}_{2}$ buffer layer is more important for the superconducting properties than the texture spread in this system. That means again GBs with angles up to $7^{\circ}$ do not obstruct the current flow. To reduce the necessary number of buffer layers, Sylva et al. recently deposited $\mathrm{Fe}(\mathrm{Se}, \mathrm{Te})$ on $\mathrm{Ni5W}$ with a single $\mathrm{CeO}_{2}$ buffer layer by pulsed laser deposition 125 . This sample showed $T_{\mathrm{c}} \sim 18 \mathrm{~K}$ (due to slight Ni poisoning) and a self-field $J_{\mathrm{c}} \sim 0.1 \mathrm{MA} / \mathrm{cm}^{2}$ as well as $J_{\mathrm{c}}(H)>20 \mathrm{kA} / \mathrm{cm}^{2}$ up to $18 \mathrm{~T}$ at $4.2 \mathrm{~K}$. 


\section{B. $A e \mathbf{F e}_{2} \mathbf{A s}_{2}(A e=\mathrm{Sr}$ and $\mathbf{B a})$ polycrystalline samples}

The Ae-122 system is one of the most actively researched FBS for magnet applications owing to its moderately high $T_{\mathrm{c}}$ (38 K for K-doped Ba-122), high $B_{\mathrm{c} 2}$ (above $50 \mathrm{~T}$ ) and small electromagnetic anisotropy ${ }^{126 \mid 127}$. In recent years, high$J_{\mathrm{c}}$ wires $\frac{128}{130}$, pancake coil tests ${ }^{131}$, and demonstration of bulk magnet trapping over $1 \mathrm{~T}^{132} 134$ have been reported. The synthesis and GB engineering guidelines for the Ae-122 system fall into two different categories: uniaxial texturing of polycrystals, and making randomly oriented polycrystals without texturing. The former and latter guidelines are similar to those for $\mathrm{Bi}_{2} \mathrm{Sr}_{2} \mathrm{Ca}_{2} \mathrm{Cu}_{3} \mathrm{O}_{10+\delta}$ tapes and $\mathrm{MgB}_{2}$, respectively. For tape wires with their flat geometry produced by the PIT method, a uniaxially aligned microstructure can be obtained relatively easily by employing plate-like Ae-122 powder and cold working. On the other hand, for untextured polycrystalline forms such as bulks or round wires, high transport $J_{\mathrm{c}}$ can be successfully achieved by grain boundary engineering, which is discussed below.

High transport $J_{\mathrm{c}}$ in Ag-clad $\mathrm{Sr}_{0.6} \mathrm{~K}_{0.4} \mathrm{Fe}_{2} \mathrm{As}_{2}$ tapes prepared by the ex-situ PIT method was reported by Zhang et al ${ }^{129}$. To produce Sr-122 powder, Sr fillings, $\mathrm{K}$ pieces, and $\mathrm{Fe}$ and As powders were mixed and heat-treated at $900^{\circ} \mathrm{C}$ for $35 \mathrm{~h}$. In order to improve the grain connectivity, 5wt\% Sn was added. After packing the powder into $\mathrm{Ag}$ tubes, drawing, flat rolling and hot pressing at $850^{\circ} \mathrm{C}$ under $30 \mathrm{MPa}$, Sr-122 tapes with $0.4 \mathrm{~mm}$ in thickness were obtained. $J_{\mathrm{c}}$ of the Sr-122 tape was over $10^{5} \mathrm{~A} / \mathrm{cm}^{2}$ in $10 \mathrm{~T}$ at $4.2 \mathrm{~K}$, and still remained at $8.4 \times 10^{4} \mathrm{~A} / \mathrm{cm}^{2}$ up to $14 \mathrm{~T}$. The high $J_{\mathrm{c}}$ in the Sr-122 tapes may be attributed to the combination of improved grain connectivity, grain texture, and inherently strong pinning. The $c$-axis orientation factor for the tape was 0.52, suggesting grain texturing after pressing. Such grain texturing is considered to alleviate weak-link behaviour at GBs and can also be achieved by high pressure sintering $135+137$ and conventional cold mechanical deformation processes $127|130| 138$ 141. Ag-based Sn binary alloys were deployed for ex-situ Ba-122 tapes as sheath material by Togano et al ${ }^{142}$. The Ag-Sn alloy has 
higher mechanical strength compared to pure Ag. Successful densification and texturing of the Ba-122 core were obtained. The Ag-Sn-sheathed Ba-122 tapes showed higher transport $J_{c}$ compared to those of Ag-sheathed tapes. The authors further pointed out the significantly improved smoothness of the interface between the sheath and Ba-122 core. These findings indicate the benefit of using high mechanical strength sheath. Surprisingly high $J_{\mathrm{c}}$ values in randomly oriented, fine-grain wires and bulks were reported by Weiss et al ${ }^{128}$. Sub-micron size Ba-122 powders were prepared by a mechanochemical reaction 143 . TEM showed that the average grain size is approximately $200 \mathrm{~nm}$. The randomly oriented polycrystalline structure contained many high-angle grain boundaries including clean and well

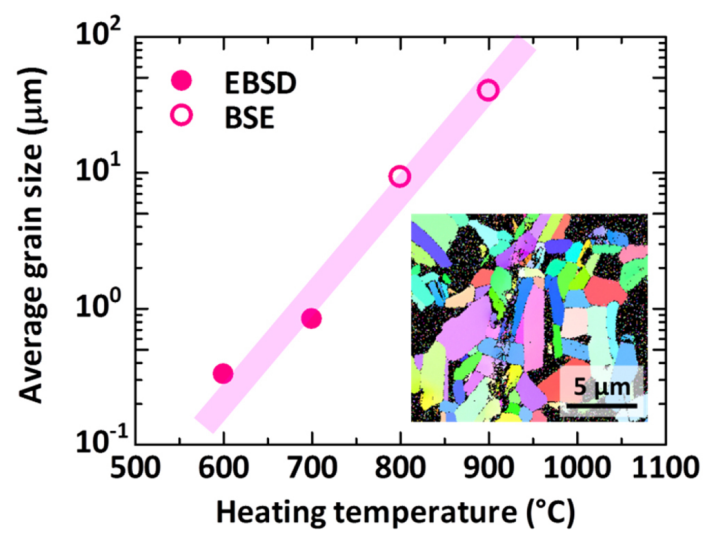

FIG. 7. Clear correlation between average grain size and processing temperature can be seen for polycrystalline Co-doped Ba-122. The inset displays the inverse pole figure map obtained by scanning electron microscope EBSD for a sample heated at $700^{\circ} \mathrm{C}$ for $48 \mathrm{~h}$. BSE stands for back-scattered electron. Reproduced from 146. 


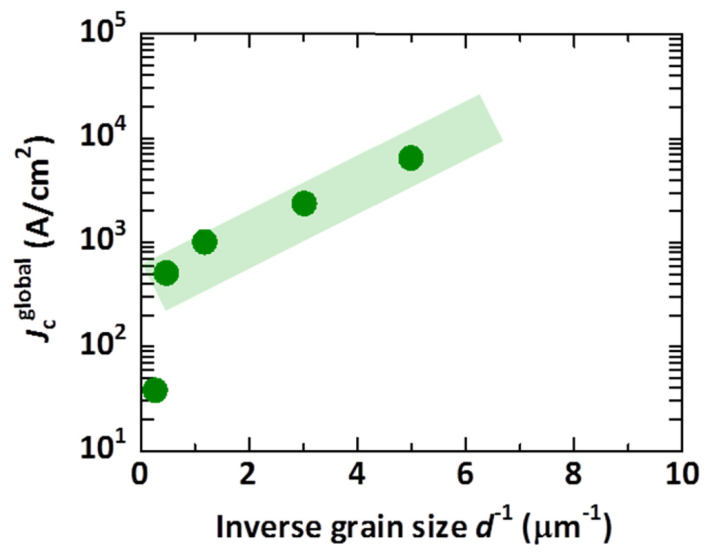

FIG. 8. Inverse grain size $(1 / d)$ dependence of the global $J_{\mathrm{c}}$ for Co-doped Ba-122 polycrystalline samples. Reproduced from 146.

connected ones. $J_{\mathrm{c}}$ at $4 \mathrm{~K}$ reached $1.2 \times 10^{5} \mathrm{~A} / \mathrm{cm}^{2}$ and $2 \times 10^{4} \mathrm{~A} / \mathrm{cm}^{2}$ for Ba-122 wires with K-doping and Co-doping, respectively. Quite recently, the additional effect by ball milling was reported by Tokuta and Yamamoto 144: high-energy ball milling introduces lattice defects in Co-doped Ba-122, resulting in high $H_{\mathrm{c} 2}$.

The influence of grain size, especially the benefit of small grains, was studied by Hecher et al. based on the Josephson junction network model ${ }^{145}$. Scanning Hall probe microscopy and magnetic hysteresis measurements on Ba-122 bulks with systematically varied grain size showed that the field dependence of untextured polycrystalline samples can be significantly reduced when the grain size is small. Shimada et al. reported on detailed microstructural investigations of the Ba122 bulk samples prepared at different heating temperatures ${ }^{146}$. A strong heating temperature dependence of the average grain size was observed for the temperature 
range between $600^{\circ} \mathrm{C}$ and $900^{\circ} \mathrm{C}$ (fig.7). Interestingly, a relationship between inverse grain size and magnetization $J_{\mathrm{c}}$ was confirmed, as shown in fig. 8 , which also supports that a small grain size would be preferable for randomly oriented polycrystalline Ba-122 samples.

Another key issue to address is chemical composition at grain boundaries. Kim et al. reported grain and grain boundary composition in K-doped Ba-122 polycrystals by atom-probe tomography 147. Significant compositional variation and segregation of oxygen impurities at grain boundaries were observed. These would suppress transport current across grain boundaries. Detailed microstructural and electromagnetic investigation on artificial grain boundaries in bicrystal and natural grain boundaries in polycrystalline samples are very helpful for grain boundary engineering in this system.

\section{DISCUSSION AND SUMMARY}

Artificial, well-defined single grain boundaries have been used for fundamental investigations such as $J_{\mathrm{c}}$ transparency as a function of mis-orientation angle $\theta_{\mathrm{GB}}$. For [001]-tilt symmetric bicrystal films, Fe(Se,Te), Co-doped Ba-122 and Nd$\mathrm{FeAs}(\mathrm{O}, \mathrm{F})$ showed the same critical angle $\theta_{\mathrm{c}}$ of around $9^{\circ}$, which is larger than for cuprates. The dominant factor for governing $\theta_{\mathrm{c}}$ may be the symmetry of the order parameter. The reduction of current-carrying cross-section of GB regions by strain effects also controls the $\theta_{c}$ as discussed by Gurevich and Pashitskii. $\frac{13}{\text {, since }}$ the sensitivity of superconductivity by strain is different for FBS and cuprates. Additionally, the local off-stoichiometry near GBs affects the size of the normal core regions 13 . Although $\theta_{\mathrm{c}}$ is different for FBS and cuprates, both superconductors showed an exponential decay of inter-grain $J_{\mathrm{c}}$ with nearly the same slope for medium angles. However, for $\theta_{\mathrm{GB}}>24^{\circ}$ the inter-grain $J_{\mathrm{c}}$ for Co-doped Ba-122 and $\mathrm{Fe}(\mathrm{Se}, \mathrm{Te})$ is almost constant, whereas such behaviour is absent for cuprates. 
Unlike YBCO, the microscopic understanding of GBs is still limited. To understand the GB characteristics, therefore, detailed microstructural analyses should be carried out to map out the local strain around the GBs. To rule out local off-stoichiometry, bicrystal experiments using stoichiometric superconductors (e.g. $\mathrm{LiFeAs}$ and $\mathrm{FeSe}$ or even $\mathrm{CaKFe}_{4} \mathrm{As}_{4}$ ) would be interesting for further studies.

Another distinct feature is that the product of the grain-boundary normal state resistance $R_{\mathrm{n}}$ and the grain boundary area $A$ for FBS is one or two orders of magnitude lower than that for cuprates due to the metallic nature of GBs (fig.9). Here, $R_{\mathrm{n}}$ corresponds to the slope of $V-I$ in the non-ohmic linear differential region and $A$ is the cross sectional area of the microbridge for transport measurements. For YBCO, band bending creates charge depletion layers around the GBs, which leads to the formation of insulating layers (Mott insulator) 16 . The presence of insulating layers at large-angle GBs was experimentally confirmed by Winkler

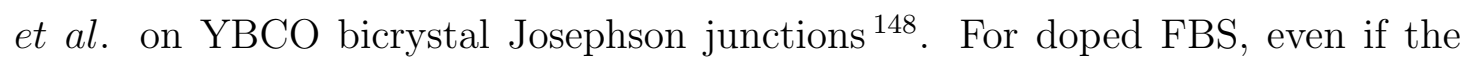
depletion layers are formed at GBs by some mechanisms like strain effects, GB regions stay in the metallic state. This may be a part of the reason why the GBs of FBS are of metallic nature.

The critical angle as well as the $\theta_{\mathrm{GB}}$ dependence of the inter-grain $J_{\mathrm{c}}$ could be different for other types of bicrystals (i.e. [010]-tilt roof and valley, and twist). Hence, various types of bicrystal experiments should be carried out in order to deeply understand the characteristics of GBs, which is very important for the applications using a polycrystalline form such as PIT wires and bulk magnets.

Regarding applications, bicrystal experiments on K-doped Ba-122, which is believed to be an important material, should be carried out. Experiments on single GBs in polycrystalline K-doped Ba-122 films have been reported ${ }^{149}$. However, the information on well-defined, single GBs is necessary for deep understanding of GB characteristics. Due to the difficulty of the thin film growth, no studies on epitaxial K-doped Ba-122 thin films have been reported to date. Very recently, we 


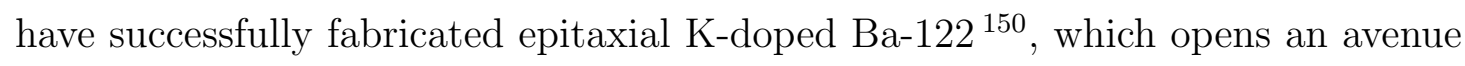
for such bicrystal experiments.

Clean GBs without wetting phase may not become obstacles for inter-grain super-currents irrespective of the mis-orientation angle, which is similar to $\mathrm{MgB}_{2}$. Even a high GB density acts as pinning centre ensemble.

From the above, grain boundary properties of FBS may be also located in between $\mathrm{MgB}_{2}$ and cuprates. Nevertheless, fundamental understanding of GB properties leads to further improvement of the current carrying capability of FBS by GB engineering.

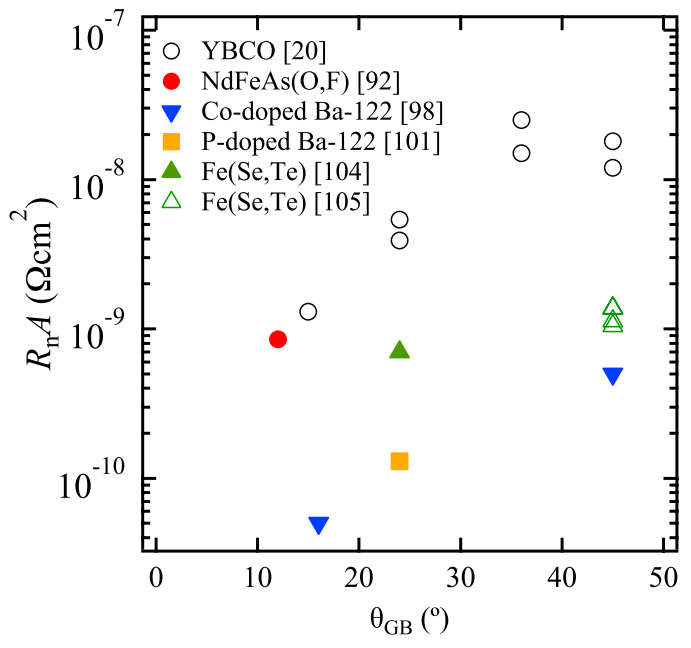

FIG. 9. Comparative $\theta_{\mathrm{GB}}$ dependence of resistance area product $R_{\mathrm{n}} A$ at low temperature for $\mathrm{YBCO}$ at $4.2 \mathrm{~K}^{20}, \operatorname{NdFeAs}(\mathrm{O}, \mathrm{F})$ at $4.2 \mathrm{~K}^{92}, \mathrm{Ba}\left(\mathrm{Fe}_{1-x} \mathrm{Co}_{x}\right)_{2} \mathrm{As}_{2}$ (Co-doped Ba-122 at $\left.4 \mathrm{~K}^{\sqrt[98]{9}}\right), \mathrm{BaFe}_{2}\left(\mathrm{As}_{1-x} \mathrm{P}_{x}\right)_{2}$ (P-doped Ba-122 at $\left.2 \mathrm{~K}^{[101}\right)$, and $\mathrm{Fe}(\mathrm{Se}, \mathrm{Te})$ at $4.2 \mathrm{~K}^{104[105}$. 


\section{ACKNOWLEDGMENTS}

This work was supported by the JSPS Grant-in-Aid for Scientific Research (B) Grant Number 16H04646 as well as JST CREST Grant Number JPMJCR18J4.

\section{REFERENCES}

${ }^{1}$ Tomimoto K, Terasaki I, Rykov A I, Miura T and Tajima S, 1999 Phys. Rev. B 60114

${ }^{2}$ Tsuei C C and Kirtley J R 2000 Rev. Mod. Phys. 72969

${ }^{3}$ Hilgenkamp H and Mannhart J 2002 Rev. Mod. Phys. 74485

${ }^{4}$ Putti M, Pallecchi I, Bellingeri E, Cimberle M R, Tropeano M, Ferdeghini C, Palenzona A, Tarantini C, Yamamoto A, Jiang J, Jaroszynski J, Kametani F, Abraimov D, Polyanskii A, Weiss J D, Hellstrom E E, Gurevich A, Larbalestier D C, Jin R, Sales B C, Sefat A S, McGuire M A, Mandrus D, Cheng P, Jia Y, Wen H H, Lee S and Eom C B, 2010 Supercond. Sci. Technol. 23034003

${ }^{5}$ Chan Siu-Wai 1994 J. Phys. Chem. Solids 551415

${ }^{6}$ Durrell J H and Rutter N A 2009 Supercond. Sci. Technol. 22013001

${ }^{7}$ Durrell J H, Eom C-B, Gurevich A, Hellstrom E E, Tarantini C, Yamamoto A and Larbalestier D C 2011 Rep. Prog. Phys. 74124511

${ }^{8}$ Hänisch J and Iida K, Superconductivity From Materials Science to Practical Applications (Springer), Chap. 10, pp. 269-302.

${ }^{9}$ Seuntjens J M and Larbalestier D C 1990 J. Appl. Phys. 672007

${ }^{10}$ Holzapfel B, Verebelyi D, Cantoni C, Paranthaman M, Sales B, Feenstra R, Christen D and Norton D P 2000 Physica C 341-348 1431

${ }^{11}$ Schoop U, Kleefisch S, Meyer S, Marx A, Alff L, Gross R, Naito M and Sato H 1999 IEEE. Trans. Appl. Supercond. 93409

${ }^{12}$ Hänisch J, Attenberger A, Holzapfel B and Schultz L 2002 Phys. Rev. B 65 052507 
${ }^{13}$ Gurevich A and Pashitskii E A 1998 Phys. Rev. B 5713878

${ }^{14}$ Song X, Daniels G, Feldmann D M, Gurevich A and Larbalestier D 2005 Nat. Mater. 4470

${ }^{15}$ Deutscher G 2010 Appl. Phys. Lett. 96122502

${ }^{16}$ Mannhart J and Hilgenkamp H 1997 Supercond. Sci. Technol. 10880

${ }^{17}$ Kasatkin A, Tsvetkovskii V and Borisenko P 2013 Universal Journal of Physics and Application 1144

${ }^{18}$ Graser S, Hirschfeld P J, Kopp T, Guster R, Andersen B M and Mannhart J 2010 Nat. Phys. 6609

${ }^{19}$ Schmehl A, Goetz B, Schulz R R, Schneider C W, Bielefeldt H, Hilgenkamp H and Mannhart J 1999 EPL 47110

${ }^{20}$ Hilgenkamp H and Mannhart J 1998 Appl. Phys. Lett. 73265

${ }^{21}$ Daniels G A, Gurevich A and Larbalestier D C 2000 Appl. Phys. Lett. 773251

${ }^{22}$ Díaz A, Mechin L, Berghuis P and Evetts J E 1998 Phys. Rev. B 58 R2960

${ }^{23}$ Hammerl G, Schmehl A, Schulz R R, Goetz B, Bielefeldt H, Schneider C W, Hilgenkamp H and Mannhart J 2000 Nature 407162

${ }^{24}$ Fâte A and Senatore C 2018 Sci. Rep. 817703

${ }^{25}$ Yuan H, Shimotani H, Tsukazaki A, Ohtomo A, Kawasaki M and Iwasa Y 2009 Adv. Funct. Mater. 191046

${ }^{26}$ Hassan M U and Wimbush S C 2015 Phys. Status Solidi A 2122037

${ }^{27}$ Jin S, Tiefel T H, Sherwood R C, Kammlott G W and Zahurak S M 1987 Appl. Phys. Lett. 51943

${ }^{28}$ Sawano K, Morita M, Tanaka M, Sasaki T, Kimura K, Takebayashi S, Kimura M and Miyamoto K 1991 Jpn. J. Appl. Phys. 30 L1157

${ }^{29}$ Durrell J H, Dennis A R, Jaroszynski J, Ainslie M D, Palmer K G B, Shi YH, Campbell A M, Hull J, Strasik M, Hellstrom E E and Cardwell D A 2014 Supercond. Sci. Technol. 27082001

${ }^{30}$ Patel A, Baskys A, Mitchell-Williams T, McCaul A, Coniglio W, Hänisch J, 
Lao M and Glowacki B A 2018 Supercond. Sci. Technol. 31 09LT01

${ }^{31}$ Durrell J H, Ainslie M D, Zhou D, Vanderbemden P, Bradshaw T, Speller S, Filipenko M and Cardwell D A 2018 Supercond. Sci. Technol. 31103501

${ }^{32}$ Dam B, Huijbregtse J M, Klaassen F C, van der Geest R C F, Doornbos G, Rector J H, Testa A M, Freisem S, Martinez J C, Stäuble-Pümpin B and Griessen R 1999 Nature 399439

${ }^{33}$ Iijima Y, Tanabe N, Ikeno Y and Kohno O 1991 Physica C 185-189 1959

${ }^{34}$ Goyal A, Norton D P, Budai J D, Paranthaman M, Specht E D, Kroeger D M, Christen D K, He Q, Saffian B, List F A, Lee D F, Martin P M, Klabunde C E, Hartfield E and Sikka V K 1996 Appl. Phys. Lett. 691795

${ }^{35}$ Buzea C and Yamashita T 2001 Supercond. Sci. Technol. 14 R115

${ }^{36}$ Larbalestier D C, Cooley L D, Rikel M O, Polyanskii A A, Jiang J, Patnaik S, Cai X Y, Feldmann D M, Gurevichi A, Squitieri A A, Naus M T, Eom C B, Hellstrom E E, Cava R J, Regan K A, Rogado N, Hayward M A, He T, Slusky J S, Khalifah P, Inumaru K and Haas M 2001 Nature 410186

${ }^{37}$ Dhallé M, Toulemonde P, Beneduce C, Musolino N, Decroux M and Flükiger R 2001 Physica $C 363155$

${ }^{38}$ Glowacki B A, Majoros M, Vickers M, Evetts J E, Shi Y and Mcdougall I 2001 Supercond. Sci. Technol. 14193

${ }^{39}$ Grasso G, Malagoli A, Ferdeghini C, Roncallo S, Braccini V, Siri A S and Cimberle M R 2001 Appl. Phys. Lett. 79230

${ }^{40}$ Kumakura H, Matsumoto A, Fujii H and Togano K 2001 Appl. Phys. Lett. 79 2435

${ }^{41}$ Kitaguchi H, Matsumoto A, Kumakura H, Doi T, Yamamoto H, Saitoh K, Sosiati H and Hata S 2004 Appl. Phys. Lett. 852842

${ }^{42}$ Martínez E, Mikheenko P, Martínez-López M, Millán A, Bevan A and Abell J S 2007 Phys. Rev. B 75134515

${ }^{43}$ Collings E W, Sumption M D, Bhatia M, Susner M A and Bohnenstiehl S D 
2008 Supercond. Sci. Technol. 21103001

${ }^{44}$ Matsushita T, Kiuchi M, Yamamoto A, Shimoyama J-I and Kishio K 2008 Supercond. Sci. Technol. 21015008

${ }^{45}$ Senkowicz B J, Mungall R J, Zhu Y, Jiang J, Voyles P M, Hellstrom E E and Larbalestier D C 2008 Supercond. Sci. Technol. 21035009

${ }^{46}$ Rosová A, Hušek I, Kulich M, Melišek T, Kováč P, Dobročka E, Kopera L, Scheiter J and Häßler W 2018 J. Alloys Compd. 764437

${ }^{47}$ Malagoli A, Braccini V, Tropeano M, Vignolo M, Bernini C, Fanciulli C, Romano G, Putti M, Ferdeghini C, Mossang E, Polyanskii A and Larbalestier D C 2008 J. Appl. Phys. 104103908

${ }^{48}$ Fujii H, Iwanade A, Kawada S and Kitaguchi H 2018 Cryogenics 8976

${ }^{49}$ Fujii H, Iwanade A and Kitaguchi H 2019 Physica C 55932

${ }^{50}$ Sugino S, Yamamoto A, Shimoyama J-I and Kishio K 2015 Supercond. Sci. Technol. 28055016

${ }^{51}$ Yamamoto A, Shimoyama J-I, Ueda S, Katsura Y, Horii S and Kishio K 2005 Supercond. Sci. Technol. 18116

${ }^{52}$ Kim J H, Dou S X, Wang J L, Shi D Q, Xu X, Hossain M S A, Yeoh W K, Choi S and Kiyoshi T 2007 Supercond. Sci. Technol. 20448

${ }^{53}$ Cai Q, Liu Y C, Ma Z Q, Yu L M and Li H J 2017 J. Mater. Sci.: Mater. 28 5645

${ }^{54}$ Cai Q, Liu Y C, Xiong J and Ma Z Q 2018 J. Mater. Sci.: Mater. 2910323

${ }^{55}$ Qaid S A S, Alzayed N S, Shahabuddin M, Ramay S M and Atiq S $2017 \mathrm{~J}$. Mater. Sci.: Mater. 2814696

${ }^{56}$ Zhao Q, Jiao C J, Zhu E L and Zhu Z 2017 J. Alloys Compd. 71719

${ }^{57}$ Cheng F, Ma Z Q, Liu C X, Li H J, Hossain M A, Bando Y, Yamaguchi Y, Fatehmulla A, Farooq W A and Liu Y C 2017 J. Alloys Compd. 7271105 ${ }^{58}$ Ma Z Q, Liu Y C, Shi Q Z, Zhao Q and Gao Z M 2008 Supercond. Sci. Technol. 21065004 
${ }^{59}$ Ma Z Q, Liu Y C, Shi Q Z, Zhao Q and Gao Z M 2009 Mater. Res. Bull. 44 531

${ }^{60} \mathrm{Ma}$ Z Q, Liu Y C and Gao Z M 2010 Scr. Mater. 63399

${ }^{61}$ Shimoyama J, Hanafusa K, Yamamoto A, Katsura Y, Horii S, Kishio K and Kumakura H 2007 Supercond. Sci. Technol. 20307

${ }^{62}$ Ozturk K, Dancer C E J, Savaskan B, Aksoy C, Guner B, Badica P, Aldica G and Celik S 2017 J. Alloys Compd. 724427

${ }^{63}$ Tachikawa K, Yamada Y, Suzuki O, Enomoto M and Aodai M 2002 Physica C 382108

${ }^{64}$ Takahashi Y, Naito T and Fujishiro H 2017 Supercond. Sci. Technol. 30125006

${ }^{65}$ Durrell J H, Dancer C E J, Dennis A, Shi Y, Xu Z, Campbell A M, Babu N H, Todd R I, Grovenor C R M and Cardwell D A 2012 Supercond. Sci. Technol. 25112002

${ }^{66}$ Fuchs G, Häßler, Nenkov K, Scheiter J, Perner O, Handstein A, Kanai T, Schultz L and Holzapfel B 2013 Supercond. Sci. Technol. 26122002

${ }^{67}$ Prikhna T A, Eisterer M, Rindfleisch M, Ortino M, Kozyrev A V, Shaternik A V, Shaternik V E, Tomsic M, Moshchil V E, Karpets M V, Sverdun V B, Ponomaryov S S, Romaka V V and Seidel P 2019 IEEE. Trans. Appl. Supercond. 296200905

${ }^{68}$ Yamada Y, Ohuchi H, Yamamoto A and Kishio K 2016 J. Japan Inst. Met. Mater. 80457

${ }^{69}$ Aldica G, Burdusel M, Popa S, Enculescu M, Pasuk I and Badica P 2015 Physica C 519184

${ }^{70}$ Badica P, Burdusel M, Popa S, Pasuk I, Ivan I, Borodianska H, Vasylkiv O, Kuncser A, Ionescu A M, Miu L and Aldica G 2016 Supercond. Sci. Technol. 29105020

${ }^{71}$ Badica P, Batalu D, Burdusel M, Grigoroscuta M A, Aldica G V, Enculescu M, Gabor R A, Wang Z Y, Huang R X and Li P F 2018 Ceram. Int. 4410181 
${ }^{72}$ Berger K, Koblischka M R, Douine B, Noudem J, Bernstein P, Hauet T and Lévêque J 2016 IEEE. Trans. Appl. Supercond. 266801005

${ }^{73}$ Murakami A, Iwamoto A and Noudem J G 2018 IEEE. Trans. Appl. Supercond. 288400204

${ }^{74}$ Häßler W, Scheiter J, Hädrich P, Kauffmann-Weiss S, Holzapfel B, Oomen M and Nielsch K 2018 Physica C 55148

${ }^{75}$ Giunchi G, Ripamonti G, Perini E, Cavallin T and Bassani E 2007 IEEE. Trans. Appl. Supercond. 172761

${ }^{76}$ Giunchi G 2011 IEEE. Trans. Appl. Supercond. 211564

${ }^{77}$ Ueda S, Shimoyama J-I, Iwayama I, Yamamoto A, Katsura Y, Horii S and Kishio K 2005 Appl. Phys. Lett. 86222502

${ }^{78}$ Togano K, Hur J M, Matsumoto A and Kumakura H 2009 Supercond. Sci. Technol. 22015003

${ }^{79}$ Li G Z, Sumption M D, Susner M A, Yang Y, Reddy K M, Rindfleisch M A, Tomsic M J, Thong C J and Collings E W 2012 Supercond. Sci. Technol. 25 115023

${ }^{80}$ Bhagurkar A G, Yamamoto A, Anguilano L, Dennis A R, Durrell J H, Babu N H and Cardwell D A 2016 Supercond. Sci. Technol. 29035008

${ }^{81}$ Bhagurkar A G, Yamamoto A, Dennis A R, Durrell J H, Aljohani T A, Babu N H and Cardwell D A 2017 J. Am. Ceram. Soc. 1002451

${ }^{82}$ Bhagurkar A G, Yamamoto A, Wang L, Xia M, Dennis A R, Durrell J H, Aljohani T A, Babu N H and Cardwell D A 2018 Sci. Rep. 813320

${ }^{83}$ Gozzelino L, Gerbaldo R, Ghigo G, Laviano F, Torsello D, Bonino V, Truccato M, Batalu D, Grigoroscuta M A, Burdusel M, Aldica G V and Badica P 2019 Supercond. Sci. Technol. 32034004

${ }^{84}$ Savaşkan B, Koparan E T, Güner S B, Öztürk K and Çelik Ş 2019 J. Supercond. Magn. 32827

${ }^{85}$ Yamamoto A, Jiang J, Tarantini C, Craig N, Polyanskii A A, Kametani F, 
Hunte F, Jaroszynski J, Hellstrom E E, Larbalestier D C, Jin R, Sefat A S, McGuire M A, Sales B C, Christen D K and Mandrus D 2008 Appl. Phys. Lett. 92252501

${ }^{86}$ Haindl S, Kidszun M, Kauffmann A, Nenkov K, Kozlova N, Freudenberger J, Thersleff T, Hänisch J, Werner J, Reich E, Schultz L and Holzapfel B Phys. Rev. Lett. 104077001

${ }^{87}$ Kametani F, Polyanskii A A, Yamamoto A, Jiang J, Hellstrom E E, Gurevich A, Larbalestier D C, Ren Z A, Yang J, Dong X L, Lu W and Zhao Z X 2008 Supercond. Sci. Technol. 22015010

${ }^{88}$ Senatore C, Flükiger R, Cantoni M, Wu G, Liu R H and Chen X H 2008 Phys. Rev. B 78054514

${ }^{89}$ Yamamoto A, Polyanskii A A, Jiang J, Kametani F, Tarantini C, Hunte F, Jaroszynski J, Hellstrom E E, Lee P J, Gurevich A, Larbalestier D C, Ren Z A, Yang J, Dong X L, Lu W and Zhao Z X 2008 Supercond. Sci. Technol. 21 095008

${ }^{90}$ Omura T, Matsumoto T, Hatano T, Iida K and Ikuta H 2018 J. Phys.: Conf. Ser. 1054012024

${ }^{91}$ Kawaguchi T, Uemura H, Ohno T, Tabuchi M, Ujihara T, Takeda Y and Ikuta H 2011 Appl. Phys. Express 4083102

${ }^{92}$ Iida K, Omura T, Matsumoto T, Hatano T and Ikuta H 2019 Supercond. Sci. Technol. 32074003

${ }^{93}$ Katase T, Hiramatsu H, Matias V, Sheehan C, Ishimaru Y, Kamiya T, Tanabe K and Hosono H 2011 Appl. Phys. Lett. 98242510

${ }^{94}$ Sato H, Hiramatsu H, Kamiya T and Hosono H 2016 Sci. Rep. 636828

${ }^{95}$ Corrales-Mendoza I, Labias-Romero J, Castillo N and Conde-Gallardo A 2019 Supercond. Sci. Technol. 32055005

${ }^{96}$ Matsumoto J, Hanzawa K, Sasase M, Haindl S, Katase T, Hiramatsu H and Hosono H 2019 Phys. Rev. Materials 3103401 
${ }^{97}$ Lee S, Jiang J, Weiss J D, Folkman C M, Bark C W, Tarantini C, Xu A, Abraimov D, Polyanski A, Nelson C T, Zhang Y, Baek S H, Jang H W, Yamamoto A, Kametani F, Pan X Q, Hellstrom E E, Gurevich A, Eom C B and Larbalestier D C 2009 Appl. Phys. Lett. 95212505

${ }^{98}$ Katase T, Ishimaru Y, Tsukamoto A, Hiramatsu H, Kamiya T, Tanabe K and Hosono H 2011 Nat. Commun. 2409

${ }^{99}$ Hiramatsu H, Katase T, Ishimaru Y, Tsukamoto A, Kamiya T, Tanabe K and Hosono H 2012 Mater. Sci. Eng., B 177515

${ }^{100}$ Iida K, Haindl S, Kurth F, Hänisch J, Schultz L and Holzapfel B 2013 Physics Procedia 45189

${ }^{101}$ Sakagami A, Kawaguchi T, Tabuchi M, Ujihara T, Takeda Y and Ikuta H 2013 Physica C 494181

${ }^{102}$ Eisterer M 2019 Phys. Rev. B 99094501

${ }^{103}$ Hänisch J, Iida K, Kurth F, Thersleff T, Trommler S, Reich E, Hühne R, Schultz L and Holzapfel B 2014 AIP Conference Proceedings 1574260

${ }^{104}$ Sarnelli E, Adamo M, Nappi C, Braccini V, Kawale S, Bellingeri E and Ferdeghini C 2014 Appl. Phys. Lett. 104162601

${ }^{105}$ Si W, Zhang C, Shi X, Ozaki T, Jaroszynski J and Li Q 2015 Appl. Phys. Lett. 106032602

${ }^{106}$ Sarnelli E, Nappi C, Camerlingo C, Enrico E, Bellingeri E, Kawale S, Braccini V, Leveratto A and Ferdeghini C 2017 IEEE. Trans. Appl. Supercond. 277400104 ${ }^{107}$ Gross P, Chaudhari P, Dimos D, Gupta A and Kogan G 1990 Phys. Rev. Lett. 64228

${ }^{108}$ Iida K, Kurth F, Chihara M, Sumiya N, Grinenko V, Ichinose A, Tsukada I, Hänisch J, Matias V, Hatano T, Holzapfel B and Ikuta H 2014 Appl. Phys. Lett. 105172602

${ }^{109}$ Zhang Q, Zhang X, Yao C, Huang H, Wang D, Dong C, Ma Y, Ogino H and Awaji S 2017 Supercond. Sci. Technol. 30065004 
${ }^{110}$ Tarantini C, Iida K, Hänisch J, Kurth F, Jaroszynski J, Sumiya N, Chihara M, Hatano T, Ikuta H, Schmidt S, Seidel P, Holzapfel B and Larbalestier D C 2016 Sci. Rep. 636047

${ }^{111}$ Guo Z, Saito H, Kondo K, Matsumoto T, Hatano T, Iida K, Hänisch J, Ikuta $\mathrm{H}$ and Hata $\mathrm{S}$, in preparation

${ }^{112}$ Iida K, Hänisch J, Trommler S, Matias V, Haindl S, Lucas del Pozo I, Hühne R, Kidszun M, Engelmann J, Schultz L and Holzapfel B 2011 Appl. Phys. Express 4013103

${ }^{113}$ Trommler S, Hänisch J, Matias V, Hühne R, Reich E, Iida K, Haindl S, Schultz L and Holzapfel B 2012 Supercond. Sci. Technol. 25084019

${ }^{114}$ Xu Z, Yuan P, Fan F and Ma Y 2018 Supercond. Sci. Technol. 31055001

${ }^{115}$ Gurevich A, Rzchowski M S, Daniels G, Patnaik S, Hinaus B M, Carillo F, Tafuri F and Larbalestier D C 2002 Phys. Rev. Lett. 88097001

${ }^{116}$ Horide T, Matsumoto K, Ichinose A, Mukaida M, Yoshida Y and Horii S 2007 Phys. Rev. B 75020504

${ }^{117}$ Palau A, Puig T, Obradors X, Feenstra R and Gapud A A 2006 Appl. Phys. Lett. 88122502

${ }^{118}$ Iida K, Sato H, Tarantini C, Hänisch J, Jaroszynski J, Hiramatsu H, Holzapfel B and Hosono H 2017 Sci. Rep. 739951

${ }^{119}$ Rodríguez O and Mariño A 2015 Physica C 5139

${ }^{120}$ Kametani F, Li P, Abraimov D, Polyanskii A A, Yamamoto A, Jiang J, Hellstrom E E, Gurevich A, Larbalestier D C, Ren Z A, Yang J, Dong X L, Lu W and Zhao Z X 2009 Appl. Phys. Lett. 95142502

${ }^{121}$ Hosono H, Tanabe K, Takayama-Muromachi E, Kageyama H, Yamanaka S, Kumakura H, Nohara M, Hiramatsu H and Fujitsu S 2015 Sci. Technol. Adv. Mater. 16033503

${ }^{122} \mathrm{Si}$ W, Zhou J, Jie Q, Dimitrov I, Solovyov V, Johnson P D, Jaroszynski J, Matias V, Sheehan C and Li Q 2011 Appl. Phys. Lett. 98262509 
${ }^{123}$ Xu Z, Yuan P, Ma Y and Cai C 2017 Supercond. Sci. Technol. 30035003

${ }^{124} \mathrm{Si}$ W, Han S, Shi X, Ehrlich S N, Jaroszynski J, Goyal A and Li Q 2013 Nat. Commun. 41347

${ }^{125}$ Sylva G, Augieri A, Mancini A, Rufoloni A, Vannozzi A, Celentano G, Bellingeri E, Ferdeghini C, Putti M and Braccini V 2019 Supercond. Sci. Technol. 32 084006

${ }^{126}$ Hosono H, Yamamoto A, Hiramatsu H and Ma Y 2018 Materials Today 21278 ${ }^{127}$ Yao C and Ma Y W 2019 Supercond. Sci. Technol. 32023002

${ }^{128}$ Weiss J D, Tarantini C, Jiang J, Kametani F, Polyanskii A A, Larbalestier D C and Hellstrom E E 2012 Nat. Mater. 11682

${ }^{129}$ Zhang X P, Yao C, Lin H, Cai Y, Chen Z, Li J Q, Dong C H, Zhang Q J, Wang D L, Ma Y W, Oguro H, Awaji S and Watanabe K 2014 Appl. Phys. Lett. 104 202601

${ }^{130}$ Gao Z S, Togano K, Matsumoto A and Kumakura H 2014 Sci. Rep. 44065

${ }^{131}$ Wang D L, Zhang Z, Zhang X P, Jiang D H, Dong C H, Huang H, Chen W G, Xu Q J and Ma Y W 2019 Supercond. Sci. Technol. 32 04LT01

${ }^{132}$ Weiss J D, Yamamoto A, Polyanskii A A, Richardson R B, Larbalestier D C and Hellstrom E E 2015 Supercond. Sci. Technol. 28112001

${ }^{133}$ Iida K 2015 Supercond. Sci. Technol. 28120501

${ }^{134}$ Ainslie M D, Yamamoto A, Fujishiro H, Weiss J D, Hellstrom E E 2017 Supercond. Sci. Technol. 30105009

${ }^{135}$ Pyon S, Suwa T, Tamegai T, Takano K, Kajitani H, Koizumi N, Awaji S, Zhou N and Shi Z X 2018 Supercond. Sci. Technol. 31055016

${ }^{136}$ Pyon S, Tsuchiya Y, Inoue H, Koizumi N, Kajitani H and Tamegai T 2014 Physica C $\mathbf{5 0 4} 69$

${ }^{137}$ Tamegai T, Pyon S, Tsuchiya Y, Inoue H, Koizumi N and Kajitani H 2015 IEEE. Trans. Appl. Supercond. 257300504

${ }^{138}$ Togano K, Gao Z, Matsumoto A and Kumakura H 2013 Supercond. Sci. Technol. 
26115007

${ }^{139}$ Huang H, Yao C, Dong C H, Zhang X P, Wang D L, Cheng Z, Li J Q, Awaji S, Wen H H and Ma Y W 2018 Supercond. Sci. Technol. 31015017

${ }^{140}$ Liu S F, Cheng Z, Yao C, Dong C H, Wang D L, Huang H, Li L, Xu G X, Zhu Y C, Liu F, Liu H J and Ma Y W 2019 Supercond. Sci. Technol. 32044007

${ }^{141}$ Xu G X, Zhang X P, Yao C, Huang H, Zhu Y C, Li L, Cheng Z, Liu S F, Huang S Y and Ma Y W 2019 Physica C 56130

${ }^{142}$ Togano K, Gao Z, Matsumoto A, Kikuchi A and Kumakura H 2017 Supercond. Sci. Technol. 30015012

${ }^{143}$ Weiss J D, Jiang J, Polyanskii A A and Hellstrom E E 2013 Supercond. Sci. Technol. 26074003

${ }^{144}$ Tokuta S and Yamamoto A 2019 APL Mater. 7111107

${ }^{145}$ Hecher J, Baumgartner T, Weiss J D, Tarantini C, Yamamoto A, Jiang J, Hellstrom E E, Larbalestier D C and Eisterer M 2016 Supercond. Sci. Technol. 29025004

${ }^{146}$ Shimada Y, Yamamoto A, Hayashi Y, Kishio K, Shimoyama J-I, Hata S and Konno T J 2019 Supercond. Sci. Technol. 32084003

${ }^{147}$ Kim Y-J, Weiss J D, Hellstrom E E, Larbalestier D C and Seidman D N 2014 Appl. Phys. Lett. 105162604

${ }^{148}$ Winkler D, Zhang Y M, Nilsson P Å, Stepantsov E A and Claeson T 1994 Phys. Rev. Lett. 721260

${ }^{149}$ Hong S-H, Lee N, Kang W and Lee S-G 2014 Supercond. Sci. Technol. 27055007 ${ }^{150}$ Qin D, Iida K, Naito M and Yamamoto A 2019 Growth of Thin Films of $\mathrm{Ba}_{1-x} \mathrm{~K}_{x} \mathrm{Fe}_{2} \mathrm{As}_{2}$ by $\mathrm{MBE}$ The 80th JSAP Autumn Meeting https://confit.atlas.jp/guide/event/jsap2019a/subject/19p-C213-14/advanced 